IIIIIII Open Access Articles

\title{
Design, Identification, and Sensitivity Analysis for Patient Preference Trials
}

The MIT Faculty has made this article openly available. Please share how this access benefits you. Your story matters.

\begin{tabular}{|l|l|}
\hline As Published & $10.1080 / 01621459.2019 .1585248$ \\
\hline Publisher & Informa UK Limited \\
\hline \hline Version & Author's final manuscript \\
\hline Citable link & https://hdl.handle.net/1721.1/132193 \\
\hline Terms of Use & Creative Commons Attribution-Noncommercial-Share Alike \\
\hline Detailed Terms & http://creativecommons.org/licenses/by-nc-sa/4.0/ \\
\hline
\end{tabular}




\title{
Design, Identification, and Sensitivity Analysis for
}

\section{Patient Preference Trials*}

\author{
Dean Knox $^{\dagger} \quad$ Teppei Yamamoto Matthew A. Baum $^{\S} \quad$ Adam J. Berinsky
}

First Draft: July 20, 2014

This Draft: February 11, 2019

Forthcoming in the Journal of the American Statistical Association

${ }^{*}$ We are grateful to Donald Green and David Nickerson for their helpful comments and suggestions. We also thank participants at the 2014 Society for Political Methodology Summer Meeting, MacMillan-CSAP Workshop at Yale University, and the 3rd ISAT/TSE Conference in Political Economy/Political Economy in Toulouse for their feedback. We acknowledge financial support from the National Science Foundation (SES-1528487 and Graduate Research Fellowship under Grant No. 1122374).

$\dagger$ Assistant Professor, Department of Politics, Princeton University, Princeton, NJ 08544. Email: dcknox@princeton.edu, URL: www.dcknox.com

${ }^{\ddagger}$ Associate Professor, Department of Political Science, Massachusetts Institute of Technology, 77 Massachusetts Avenue, Cambridge, MA 02139. Email: teppei@mit.edu, URL: http://web.mit.edu/teppei/www

${ }^{\S}$ Professor, John F. Kennedy School of Government, Harvard University.

"Professor, Department of Political Science, Massachusetts Institute of Technology, 77 Massachusetts Avenue, Cambridge, MA 02139. 


\begin{abstract}
Social and medical scientists are often concerned that the external validity of experimental results may be compromised because of heterogeneous treatment effects. If a treatment has different effects on those who would choose to take it and those who would not, the average treatment effect estimated in a standard randomized controlled trial (RCT) may give a misleading picture of its impact outside of the study sample. Patient preference trials (PPTs), where participants' preferences over treatment options are incorporated in the study design, provide a possible solution. In this paper, we provide a systematic analysis of PPTs based on the potential outcomes framework of causal inference. We propose a general design for PPTs with multi-valued treatments, where participants state their preferred treatments and are then randomized into either a standard RCT or a self-selection condition. We derive nonparametric sharp bounds on the average causal effects among each choice-based subpopulation of participants under the proposed design. We also propose a sensitivity analysis for the violation of the key ignorability assumption sufficient for identifying the target causal quantity. The proposed design and methodology are illustrated with an original study of partisan news media and its behavioral impact. (194 words)
\end{abstract}

Key Words: randomized controlled trial, external validity, causal inference, nonparametric bounds 


\section{Introduction}

Randomized controlled trials (RCTs) are widely used in the social and medical sciences to estimate the causal effects of treatments of interest. The random assignment of treatments ensures the internal validity of the study, in the sense that observed differences in the distribution of outcomes between randomized treatment groups can be interpreted as causal effects of the treatments. Carefully controlled randomization, however, often comes at the cost of external validity. That is, conclusions from RCTs may not generalize to situations outside of that particular experiment. Without sufficient external validity, RCTs are not informative about the substantive, real-world questions in which scientists and practitioners are ultimately interested.

In RCTs, preferences of experimental subjects over treatment options often play an important role. Even in a well controlled study on a representative sample from the target population, heterogeneity of treatment effects across treatment preferences may limit the study's externally validity. For example, a medical treatment that was found to be ineffective on average in a RCT may in fact be highly beneficial for the patients who would choose to take it if they were able to. In a standard RCT, however, researchers cannot make such nuanced inferences because all subjects are forced to take treatments randomly chosen by the researchers.

In this paper, we propose a new experimental design for patient preference trials (PPTs), in which subjects' preferences over treatments are systematically incorporated in the study design. The proposed design consists of two stages of randomization and synthesizes many of the variants of PPTs previously used in social (Gaines and Kuklinski, 2011; Arceneaux et al., 2012) and medical (King et al., 2005, Howard and Thornicroft, 2006) applications. First, all participants state their preferred treatments prior to entering the study. Then, we randomize them into either a standard RCT or a self-selection condition. In the latter condition, they choose the treatment as they would in the real world. Finally, we measure the outcome variables of interest. The proposed design is novel in that it allows the researcher to incorporate in the analysis the discrepancy between subjects' stated preferences and their actual choice of 
treatments. This modification is important because respondents to a survey question often fail to report their underlying preferences to the interviewer, whether consciously or subconsciously.

Using the potential outcomes framework of causal inference (Neyman, 1923; Rubin, 1974), we define a causal quantity which we call the average choice-specific treatment effect (ACTE), representing the conditional average treatment effect for the subpopulation of subjects who would choose a particular treatment option. We show that the point identification of this quantity for a multi-valued treatment requires the strong assumption that the discrepancy between stated preference and actual choice is ignorable. That is, the naïve estimate of the ACTE using stated preference will be biased if the measurement errors are systematically correlated with unobserved characteristics that affect the potential outcomes.

To make valid inference about the ACTE under the proposed design, we derive nonparametric sharp bounds on this causal quantity without assuming the ignorability of the measurement error in stated treatment preferences. We also propose a sensitivity analysis where we quantify the assumed informativeness of the stated preferences about actual choices via a sensitivity parameter and analyze how the ACTE responds to the change in this parameter. Finally, we develop a simulation-based procedure to make statistical inference for the bounds and sensitivity results in small samples. We provide opensource software, ppt, for implementing the proposed methodology.

We illustrate the proposed design and methodology with an original survey experiment, where we investigate the effect of partisan political news media on the subjects' perception of the media and their subsequent political behavior. Our primary interest is in how the effects of our treatments vary depending on whether subjects would actually consume such partisan media if they could choose to do so.

Despite the prevalence of PPTs across scientific disciplines, very few methodological investigations have been conducted on the topic from the perspective of causal inference. A notable exception is Long et al. (2008), who employ a framework similar to ours to define causal quantities. For the identification and estimation of those quantities, however, they assume a parametric model between the unobserved choice and observed covariates for participants in the self-selection condition and use an EM algorithm to estimate the causal quantities as functions of model parameters. In contrast, our model-free approach 
avoids distributional or functional-form assumptions for better credibility of the resulting inference.

The rest of the paper proceeds as follows. Section 2 describes the background motivation of the empirical example. Section 3 formally describes the proposed design and defines causal quantities of interest and assumptions. Sections 4, 5 and 6 discuss the proposed methodology. Section 7 applies the method to the empirical example. Section 8 investigates finite-sample performance of the proposed inferential approach via Monte Carlo simulations. Section 9 concludes.

\section{A Motivating Example}

In recent years, many scholars (e.g., Prior, 2007) have explored the political consequences of increased media choice in the 21 st century. The explosion of media outlets has vastly increased the choices available to consumers and allowed for the development of ideological "niche" news programming (Hamilton, 2005). A great deal of research has sought to determine the effects of this unprecedented media fragmentation (e.g., Stroud, 2011; Kim, 2009; Iyengar and Hahn, 2009; Levendusky, 2013).

Among several significant strands of this research program, a predominant body of scholarship has sought to delineate the effects of consuming ideologically polarized media on attitudes towards the broader mass media. According to Gallup (2014), between 1976 and 2014, the percentage of Americans expressing "a great deal" or "a fair amount" of trust in the media fell from 72 to 44 percent. People who distrust the media may conclude it cannot report in an unbiased manner. As a result, the public may dismiss media content as unreliable and increasingly become suspicious of and antagonistic toward the news media more generally (Arceneaux et al., 2012; Ladd, 2012).

To explore this phenomenon, we conducted an experiment in June 2014 on a sample of 3,023 American adults, recruited by Survey Sampling International (SSI). Our goal was to estimate the effect of exposing subjects to pro- and counter-attitudinal political news programming (as opposed to non-political entertainment shows) on their sentiment towards specific news programs and the media in general. We also explored whether such programming produces behavioral responses, such as changes in propensity to discuss the content of the media with friends. Specifically, we selected a short clip from each of 
the following television programs: (1) The Rachel Maddow Show (MSNBC), (2) Jamie's Kitchen with Jamie Oliver (Food Network), (3) Dirty Jobs with Mike Rowe (Discovery Channel), and (4) The O’Reilly Factor with Bill O'Reilly (Fox News). We carefully selected clips from the two political shows—Rachel Maddow and The O'Reilly Factor- to match as closely to each other in topic and content as possible. We selected clips that focused on energy policy (specifically, the Obama administration's policies regarding domestic energy production and their effects on gas prices). Finally, we merged the two entertainment shows into a single treatment condition ("entertainment") in our analysis.

One of our primary concerns in the design of our study was that the existing experimental studies of partisan media effects had limited external validity because they paid inadequate attention to the preferences of subjects over treatment options. Namely, the average treatment effect obtained in a standard RCT could mask fundamental heterogeneity across different types of individuals and misrepresent the overall impact of media polarization in the "real" political world. For instance, it could be the case that partisan news is highly persuasive for some people-say, those least likely to consume it in the real world—while having little or no persuasive effect among people who are most likely to consume it.

A natural approach to incorporating preferences is to adopt one of the commonly used PPT designs. For example, Arceneaux et al. (2012) conducted a similar media choice experiment in which respondents were asked their news preferences before being randomly assigned to a particular treatment condition. A PPT based on the measurement of stated preferences like this, however, appears inadequate in our context. This is because research has shown that people often have difficulty assessing what they would actually do or prefer (Clausen, 1968) or have done in the past (Prior, 2009) when offered a hypothetical choice or asked about past behavior. Theories regarding the source of this gap between self-reported preferences and actual behavior, like media consumption, are manifold. These theories range from a bias toward offering socially desirable responses on topics like voting (Rogers and Aida, 2013) and sensitive topics (Brown and Sinclair, 1999; Hser et al., 1999; Payne, 2010); to selective retention of pro-attitudinal information (Campbell et al., 1960) or motivated reasoning (Levendusky, 2013); to an inability to accurately remember prior behavior (Tourangeau, 1999). 
Given these considerations about the inadequacy of existing experimental designs, we implemented a new PPT design which we will describe in Section 3. In Section 7 we present results from our analysis of this experiment with our proposed methodology.

\section{Design and Assumptions}

In this section, we introduce the notation required for our methodology. We define our causal quantities of interest and discuss their substantive interpretations. We then introduce several assumptions for identification analysis.

\subsection{Notation and the Proposed Design}

Suppose that we have a random sample of $N$ experimental subjects from the population of interest. We consider a study where the goal is to estimate the effect of a $J$-valued treatment on an outcome of interest. Let $A_{i} \in \mathcal{A} \equiv\{0,1, \ldots, J-1\}$ denote the treatment that subject $i$ actually receives in the study. For the rest of the paper, we call this the "actual treatment," or simply the "treatment" when the meaning is obvious from the context. Without loss of generality, we impose the standard total ordering on $\mathcal{A}$.

Our proposed design for patient preference trials proceeds as follows. First, all $N$ subjects in the study sample are asked to state their preferred treatment, $S_{i} \in \mathcal{A}$. Second, after an optional "washout" period, or a set of additional questions as we discuss in Section 7.1 , the subjects are randomized into one of the two conditions: Either they will be forced to take the randomly assigned treatment, or they will be allowed to freely choose the treatment of their own accord. We refer to this actual choice as $C_{i} \in \mathcal{A}$. Formally, we use the "design indicator" $D_{i} \in\{0,1\}$ to denote whether subject $i$ is in the forced-exposure condition $\left(D_{i}=1\right)$ or the free-choice condition $\left(D_{i}=0\right)$. Third, the subjects then receive treatment $\left(A_{i}\right)$ according to the protocol determined by their design indicator. That is, $A_{i}$ is randomized if and only if $D_{i}=1$; in this case, $C_{i}$ is unobserved. For the subjects with $D_{i}=0$, their treatments equal the treatments they have chosen. Therefore, we have $A_{i}=C_{i}$ if $D_{i}=0$. Finally, the outcome of interest, $Y_{i} \in \mathcal{Y}$, is measured for every subject. 
Under the proposed design, the potential outcome for subject $i$ can be defined as $Y_{i}(a) \in \mathcal{Y}$. This represents the value of the outcome of interest that would be realized if $i$ received the treatment $a \in$ $\mathcal{A}$. By this notation, we are implicitly making the stable unit treatment value assumption (SUTVA, Rubin, 1990), which posits that subjects cannot be affected by the treatments received by any other subjects (no interference) and that subjects exhibit the same value of the outcome no matter how the treatment $A_{i}=a$ was received (stability or consistency). In particular, the notation assumes that there is no design effect, i.e., the potential outcomes remain stable across the two design conditions. Long et al. (2008) call this assumption the exclusion restriction. The no-design-effect assumption would be violated if, for example, a nominally identical treatment had different effects on the outcome for the same unit depending on whether the treatment was randomly assigned in the forced-exposure condition or voluntarily chosen in the free-choice condition. Under the SUTVA, we can express the observed outcome as $Y_{i}=\sum_{a \in \mathcal{A}} Y_{i}(a) \mathbf{1}\left\{A_{i}=a\right\}=Y_{i}\left(A_{i}\right)$ for any $i$. The cumulative distribution function (CDF) of $Y_{i}(a)$ is denoted by $F_{Y(a)}(y)=\operatorname{Pr}\left(Y_{i}(a) \leq y\right)$.

The diagram in Figure 1 graphically summarizes the proposed design. Several important features of this design are worth mentioning. First, the proposed design combines the standard RCT ( $D_{i}=1$, upper arm) with a pure self-selection study ( $D_{i}=0$, lower arm) via randomization. As discussed in Section 4 , this allows us to infer more about the unobserved choice behavior of the subjects who are assigned to the forced-exposure condition. Second, our design clearly distinguishes the stated preference of the subjects $\left(S_{i}\right)$ from their actual choice (or "revealed preferences," as they are often called in the social sciences, $C_{i}$ ). As pointed out in Section 2, social and medical scientists are often concerned that stated preferences may be unreliable due to various sources of systematic measurement error. Thus, a "naïve" analysis that takes the stated preferences at their face value and ignores the possible measurement error may lead to an invalid estimate. Finally, note that we allow the treatment variable to be multi-valued, instead of binary. In fact, as previously shown by Long et al. (2008) and Gaines and Kuklinski (2011) and revisited in Section 4, assuming a binary treatment greatly simplifies the problem, leading to point identification of the average choice-specific treatment effects (defined shortly). However, as in the media choice example, 


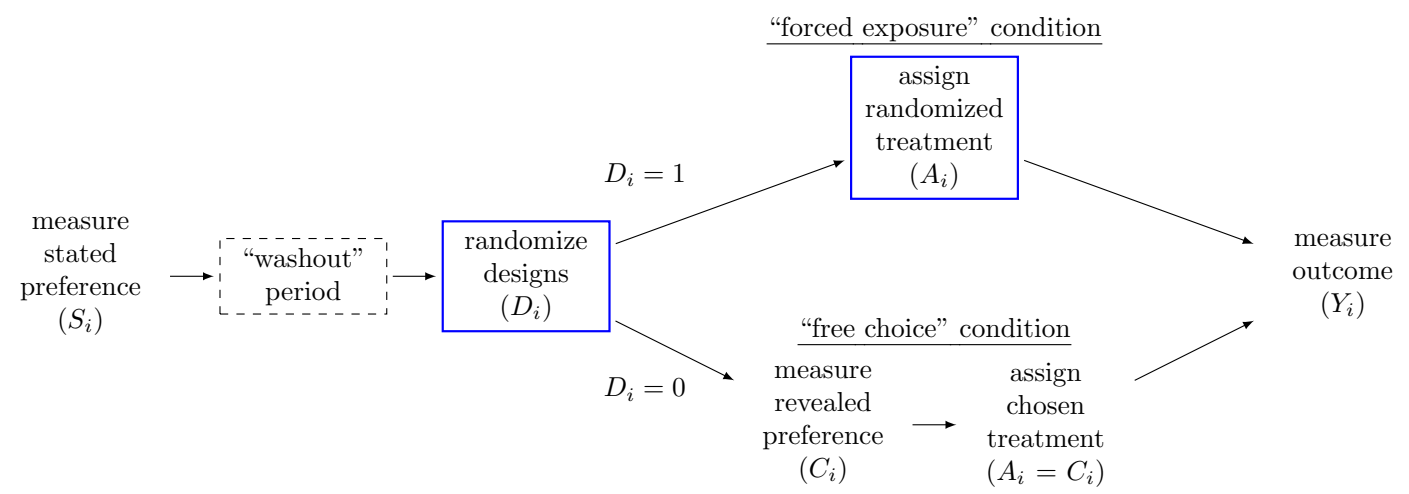

Figure 1: Diagram of the Proposed PPT Design. In the proposed design, subjects are first asked to state preferences about the treatment options $\left(S_{i}\right)$ and (after an optional "washout" period) randomized into design conditions $\left(D_{i}\right)$. In the "forced exposure" arm (top, $D_{i}=1$ ), subjects are randomly assigned to treatments irrespective of their stated preferences $\left(A_{i}\right)$. In the "free choice" arm (bottom, $D_{i}=0$ ), the subjects are asked to choose the treatment they want to take $\left(C_{i}\right)$ and actually exposed to that treatment $\left(A_{i}=C_{i}\right)$. Finally, the outcome measure is taken on all subjects $\left(Y_{i}\right)$. In the diagram, the blue boxes indicate random assignment and the dashed box indicates an optional component.

social and medical scientists are often interested in testing the effects of more than two treatments in a single study.

There exist many previous studies in both social and medical sciences that utilize PPT designs related to ours (King et al., 2005; Kowalski and Mrdjenovich, 2013). For example, a commonly used design in clinical trials (Brewin and Bradley, 1989) begins by asking subjects whether they have a preference between treatment options and then randomizing only those subjects who expressed no preference. A close variant of this design (Schmoor et al., 1996) asks participants whether they agree to be randomized into treatment conditions. These designs may be preferable in situations where randomizing subjects against their preferences is practically or ethically infeasible. However, these designs are critically limited in terms of external validity because the treatment effects are only identified for subjects who have no (or weak) treatment preferences.

The key advantage of our proposed design is the combination of stated preference measurement for all subjects and randomization into the forced-exposure and free-choice conditions, which has never been implemented as far as we are aware. For example, a popular approach that involves measurement of stated preferences at the beginning of study forces all respondents to randomly assigned treatments 
(Torgerson et al., 1996), whereas studies that contain randomization into forced-exposure and free-choice arms typically omit stated preference measurement (Gaines and Kuklinski, 2011). The importance of measuring stated preferences before randomization is worth emphasizing, for (as formally discussed in Section 4 the variable enables more informative inference across the forced-exposure and free-choice arms. For example, Arceneaux et al. (2012) report results from a series of RCTs, one of which included measurement of stated preferences and another which involved randomization into a free-choice condition. Unfortunately, these two studies are conducted separately on populations with possibly different characteristics, rendering inference from combined data difficult.

\subsection{Quantities of interest}

A common causal quantity of interest in the social and medical sciences is the (population) average treatment effect (ATE), which is defined as follows.

$$
\delta\left(a, a^{\prime}\right) \equiv \mathbb{E}\left[Y_{i}(a)-Y_{i}\left(a^{\prime}\right)\right]
$$

for any $a$ and $a^{\prime} \in \mathcal{A}$. This quantity represents the (additive) causal effect of treating a unit with treatment $a$ as opposed to treatment $a^{\prime}$, averaged unconditionally over the sampling distribution. It is widely known that the ATE can be nonparametrically identified in a standard RCT, where both treatments $a$ and $a^{\prime}$ are randomly assigned with non-zero probabilities, and can be estimated with very simple estimators such as the difference-in-means.

However, the ATE is often not the only causal parameter that is of substantive interest in a given applied setting. For example, in the media choice experiment introduced in Section 2, our interest was not only in the average effect of exposing every American adult to one program versus another, but also in investigating heterogeneity in media effects based on the respondents' likely media consumption in the real world. Likewise, in a medical application, researchers may want to study whether a new treatment has beneficial effects on the patients who would actually choose to use the treatment, or whether it may have a potential harmful impact on patients if it is applied in spite of a diverging preference. 
In the rest of this paper, we focus on an alternative causal quantity which addresses these more nuanced questions,

$$
\tau\left(a, a^{\prime} \mid c\right) \equiv \mathbb{E}\left[Y_{i}(a)-Y_{i}\left(a^{\prime}\right) \mid C_{i}=c\right]
$$

for any $a, a^{\prime}$ and $c \in \mathcal{A}$. We call this quantity the average choice-specific treatment effect (ACTE). The ACTE represents the average effect of treating a unit with treatment $a$ instead of $a^{\prime}$ among the units who would choose treatment $c$ if they were allowed to. For example, in the media choice experiment, we may be interested in the effect of watching a pro-attitudinal news program $(a)$ instead of an entertainment show $\left(a^{\prime}\right)$ among those who would actually be watching entertainment when they were freely choosing the programs to watch $\left(c=a^{\prime}\right)$. Similarly, a psychiatrist may want to estimate the potentially adverse effect of imposing a new therapy on patients who would prefer to keep to the old treatment. Thus, the ACTE is useful for the investigation of substantively meaningful heterogeneity in treatment effects in a "natural" condition, where units would be choosing treatments without an intervention from researchers. Note that, as expected, the overall ATE can be expressed as the weighted average of the ACTEs, where the weights are given by the proportions of units who would choose each of the treatment options (i.e., $\left.\delta\left(a, a^{\prime}\right)=\sum_{c} \tau\left(a, a^{\prime} \mid c\right) \operatorname{Pr}\left(C_{i}=c\right)\right)$.

The ACTE has a close connection with the more commonly used average treatment effect on the treated (ATT), defined as follows.

$$
\gamma\left(a, a^{\prime}\right) \equiv \mathbb{E}\left[Y_{i}(a)-Y_{i}\left(a^{\prime}\right) \mid A_{i}=a\right]
$$

for $a$ and $a^{\prime} \in \mathcal{A}$. The ATT represents the average effect of treatment $a$ versus $a^{\prime}$ among those units who are actually treated with $a$. Conventionally in the literature, how those units come to be actually treated with $a$ is left implicit in the definition of this quantity. For example, in a standard RCT where treatments are randomly assigned and imposed, the ATT is equivalent to the ATE because $A_{i}$ is statistically independent of the potential outcomes (i.e. $\gamma\left(a, a^{\prime}\right)=\delta\left(a, a^{\prime}\right)$ for any $\left.a, a^{\prime} \in \mathcal{A}\right)$. On the other hand, in the so-called encouragement design where an encouragement (or "instrument") for taking a particular treatment option is randomized (e.g. Hirano et al., 2000), the actual treatment status $A_{i}$ reflects the subject's 
voluntary action of choosing to take the treatment and the ATT now has a substantive meaning similar to the ACTE. This implies that the substantive interpretation of the ATT as a causal quantity crucially depends on the study design. In this paper, we opt to introduce the new causal quantity ACTE because its interpretation is clearer and less affected by auxiliary design assumptions than the ATT.

\subsection{Assumptions}

Here, we introduce a set of statistical assumptions and discuss their relationships with the design we propose. Note that the proposed design involves two random assignments. First, the randomization of subjects into the forced-exposure and free-choice conditions implies the following assumption.

\section{Assumption 1 (Randomization of Designs)}

$$
\left\{Y_{i}(a), C_{i}, S_{i}\right\} \Perp D_{i} \quad \text { for all } \quad a \in \mathcal{A} .
$$

Long et al. (2008) refer to this assumption as "no selection bias from randomization." Second, in the forced-exposure condition, the treatments are randomly assigned and imposed on each subject. This implies that the following assumption is also guaranteed to be true.

\section{Assumption 2 (Randomization of the Forced Treatment)}

$$
\left\{Y_{i}(a), C_{i}, S_{i}\right\} \Perp A_{i} \mid D_{i}=1 \text { for all } a \in \mathcal{A} \text {. }
$$

In addition to these design-guaranteed assumptions, existing studies using PPTs often make the following untestable assumption (e.g. Arceneaux et al., 2012).

\section{Assumption 3 (Mean Ignorability of Measurement Error)}

$$
\mathbb{E}\left[Y_{i}(a) \mid C_{i}=c\right]=\mathbb{E}\left[Y_{i}(a) \mid S_{i}=c\right] \quad \text { for any } \quad a, c \in \mathcal{A}
$$

This assumption states that the potential outcomes of the units who would choose a particular treatment option are on average equal to the potential outcomes of the (potentially different) set of units who state that they would choose the same treatment. In other words, Assumption 3 holds if the discrepancy 
between the stated and revealed preferences (which one may call the measurement error if the stated preference is thought of as a measure of underlying preference) is ignorable. The assumption may be violated if the discrepancy between the stated preference and actual choice is systematically correlated with any background characteristic of the units that are associated with the potential outcomes.

Assumption 3 is not directly testable because the conditional expectation on the left-hand side is unobservable for $a \neq c$. However, Assumption 3 has two empirical implications which can be tested with observed information. First, Assumptions 1, 2 and 3 jointly imply the following relationship.

$$
\mathbb{E}\left[Y_{i} \mid A_{i}=a, D_{i}=0\right]=\mathbb{E}\left[Y_{i} \mid A_{i}=S_{i}=a, D_{i}=1\right]
$$

for any $a \in \mathcal{A}$. Second, for outcomes that are bounded from below $(\underline{y})$ and above $(\bar{y})$, it can be shown that the following inequalities must hold under Assumptions 1,2 and 3 .

$$
\underline{y} \leq \frac{\mathbb{E}\left[Y_{i} \mid A_{i}=a, D_{i}=0\right]-\mathbb{E}\left[Y_{i} \mid A_{i}=S_{i}=a, D_{i}=0\right] \operatorname{Pr}\left(A_{i}=a \mid S_{i}=a, D_{i}=0\right)}{1-\operatorname{Pr}\left(A_{i}=a \mid S_{i}=a, D_{i}=0\right)} \leq \bar{y}
$$

for any $a \in \mathcal{A}$. Proofs are provided in Web Appendix A.1.

Assumption 3 may be attractive because, as we show in Section 4 , it allows the point identification of the ACTE only with the forced-exposure condition. By making Assumption 3, the researcher can save the cost of employing an additional experimental arm. However, the assumption is a strong one in many applied contexts, as we discussed in Sections 2 and 3.1. In such applications, we recommend against dropping the free-choice condition entirely, and also recommend that the above observable implications of the assumption be tested with the collected data before the assumption is made in the analysis. Tests can be conducted in the usual manner based on the sample analogues of the expressions and their asymptotic sampling properties, obtained via standard techniques like the delta method.

\section{Nonparametric Identification Analysis}

In this section, we present the results of our nonparametric identification analysis for the ACTE. Our goal is to find nonparametric bounds on $\tau\left(a, a^{\prime} \mid c\right)$ as functions of the joint distribution of $\left\{Y_{i}, D_{i}, A_{i}, S_{i}\right\}$, which are always observed for all $i \in\{1, \ldots, N\}$ under the proposed design. First, we consider the 
identifiability of the ACTE when we only make the assumptions that are guaranteed to hold by the study design (i.e. Assumptions 1 and 2) as well as the SUTVA and no design effect. In Web Appendix A.2, we show that the ACTE can be expressed as follows under those assumptions.

$$
\tau\left(a, a^{\prime} \mid c\right)=\frac{1}{\operatorname{Pr}\left(A_{i}=c \mid D_{i}=0\right)}\left\{\begin{array}{l}
\mathbb{E}\left[Y_{i} \mid A_{i}=a, D_{i}=1\right]-\mathbb{E}\left[Y_{i} \mid A_{i}=a^{\prime}, D_{i}=1\right] \\
-\mathbb{E}\left[Y_{i} \mid A_{i}=a, D_{i}=0\right] \operatorname{Pr}\left(A_{i}=a \mid D_{i}=0\right) \\
+\mathbb{E}\left[Y_{i} \mid A_{i}=a^{\prime}, D_{i}=0\right] \operatorname{Pr}\left(A_{i}=a^{\prime} \mid D_{i}=0\right) \\
-\sum_{c^{\prime} \notin\{a, c\}} \mathbb{E}\left[Y_{i}(a) \mid C_{i}=c^{\prime}\right] \operatorname{Pr}\left(A_{i}=c^{\prime} \mid D_{i}=0\right) \\
+\sum_{c^{\prime} \notin\left\{a^{\prime}, c\right\}} \mathbb{E}\left[Y_{i}\left(a^{\prime}\right) \mid C_{i}=c^{\prime}\right] \operatorname{Pr}\left(A_{i}=c^{\prime} \mid D_{i}=0\right)
\end{array}\right\},
$$

for any $a, a^{\prime}$ and $c \in \mathcal{A}$. Equation (4) immediately gives us three important results. First, equation (4) contains as many as $2(J-2)$ terms (when $a \neq a^{\prime} \neq c$ ) that cannot be identified from observed data under Assumptions 1 and 2. When $a \neq a^{\prime}=c$ or $a=c \neq a^{\prime}$, some of these can replaced with observed quantities, but $J-2$ unidentified terms remain. Thus, it can be concluded that the ACTE is unidentified by the proposed PPT design itself.

Second, when the treatment is binary as in many social and medical RCTs (i.e., $J=2$ ), the unidentified terms drop out of equation (4). This implies that the ACTE is point-identified under Assumptions 1 and 2 alone if $J=2$, and is written as follows.

$$
\tau\left(a, a^{\prime} \mid c\right)= \begin{cases}\frac{\mathbb{E}\left[Y_{i} \mid D_{i}=0\right]-\mathbb{E}\left[Y_{i} \mid A_{i}=a^{\prime}, D_{i}=1\right]}{\operatorname{Pr}\left(A_{i}=a \mid D_{i}=0\right)} & \text { if } \quad c=a, \\ \frac{\mathbb{E}\left[Y_{i} \mid A_{i}=a, D_{i}=1\right]-\mathbb{E}\left[Y_{i} \mid D_{i}=0\right]}{\operatorname{Pr}\left(A_{i}=a^{\prime} \mid D_{i}=0\right)} & \text { if } \quad c=a^{\prime},\end{cases}
$$

for $a, a^{\prime}$ and $c \in\{0,1\}$. This exactly matches Gaines and Kuklinski's (2011, p.729) result, where they consider a PPT design that is identical to ours except that it does not contain the measurement of stated preferences $S_{i}$ and only the $J=2$ case is considered. The same result is also obtained by Long et al. (2008) using a framework more similar to ours. Thus, we verify their earlier result under the current framework and also show that our proposed framework encompasses theirs as a special case.

Third, if we make Assumption 3 in addition to Assumptions 1 and 2, the unidentified terms in equation (4) become identified as $\mathbb{E}\left[Y_{i}\left(a^{\prime \prime}\right) \mid C_{i}=c^{\prime}\right]=\mathbb{E}\left[Y_{i} \mid A_{i}=a^{\prime \prime}, S_{i}=c^{\prime}, D_{i}=1\right]$ for $a^{\prime \prime} \in\left\{a, a^{\prime}\right\}$. This implies that the ACTE can be point identified for any $J$ under Assumptions 1,2 and 3 and is given by 
the following expression.

$$
\tau\left(a, a^{\prime} \mid c\right)=\mathbb{E}\left[Y_{i} \mid S_{i}=c, A_{i}=a, D_{i}=1\right]-\mathbb{E}\left[Y_{i} \mid S_{i}=c, A_{i}=a^{\prime}, D_{i}=1\right]
$$

for $a, a^{\prime}$ and $c \in \mathcal{A}$. Equation (5) makes it clear that the forced-exposure group alone is sufficient for the identification of the ACTE when we make Assumptions 2 and 3 . Indeed, Arceneaux et al. (2012, pp.182-3) use equation (5) to estimate the ACTE in their experiment, which consisted of the forcedexposure arm of our proposed design alone. As we discussed in Section 3, while this design choice may be reasonable in some applied contexts, it must be made with caution because Assumption 3 is strong and omitting the free-choice condition precludes the testing of its observable implications. From here on, we call equation (5) the "naïve estimator" of the ACTE.

What if we want to avoid Assumption 3 or analyze non-binary treatments? In this case, the forcedexposure arm alone is completely uninformative about the ACTE. We present two partial identification results using both forced-exposure and free-choice arms, which provide sharp bounds (i.e., the tightest possible given all the observed information; Manski, 1995) on $\tau\left(a, a^{\prime} \mid c\right)$ under various scenarios.

\subsection{General Results}

Our first set of results, summarized in Proposition 1, is the more general of the two and valid for any real-valued outcome $(\mathcal{Y} \subseteq \mathbb{R})$ under the proposed design.

Proposition 1 (Nonparametric Sharp Bounds on the ACTE) Under Assumptions 1 and $2, \tau\left(a, a^{\prime} \mid c\right)$ can be partially identified at least up to the following nonparametric bounds:

$$
\begin{gathered}
\sum_{s \in \mathcal{A}}\left(\operatorname { l i m } _ { y ^ { * } \rightarrow - \infty } \left[\int_{y^{*}}^{\infty} \max \left\{0,1-\frac{1-F\left(y \mid s, a^{\prime}, 1\right)+\left\{1-F\left(y \mid s, a^{\prime}, 0\right)\right\} P\left(a^{\prime} \mid s, 0\right)}{\operatorname{Pr}\left(A_{i}=c \mid S_{i}=s, D_{i}=0\right)}\right\}\right.\right. \\
\left.\left.-\min \left\{1, \frac{F(y \mid s, a, 1)-F(y \mid s, a, 0) P(a \mid s, 0)}{\operatorname{Pr}\left(A_{i}=c \mid S_{i}=s, D_{i}=0\right)}\right\} \mathrm{d} y\right]\right) \operatorname{Pr}\left(S_{i}=s \mid A_{i}=c, D_{i}=0\right) \\
\leq \tau\left(a, a^{\prime} \mid c\right) \leq \\
\sum_{s \in \mathcal{A}}\left(\operatorname { l i m } _ { y ^ { * } \rightarrow - \infty } \left[\int_{y^{*}}^{\infty} \min \left\{1, \frac{F\left(y \mid s, a^{\prime}, 1\right)-F\left(y \mid s, a^{\prime}, 0\right) P\left(a^{\prime} \mid s, 0\right)}{\operatorname{Pr}\left(A_{i}=c \mid S_{i}=s, D_{i}=0\right)}\right\}\right.\right. \\
\left.\left.-\max \left\{0,1-\frac{1-F(y \mid s, a, 1)+\{1-F(y \mid s, a, 0)\} P(a \mid s, 0)}{\operatorname{Pr}\left(A_{i}=c \mid S_{i}=s, D_{i}=0\right)}\right\} \mathrm{d} y\right]\right) \operatorname{Pr}\left(S_{i}=s \mid A_{i}=c, D_{i}=0\right)
\end{gathered}
$$


where $F(y \mid s, a, d)=\operatorname{Pr}\left(Y_{i} \leq y \mid S_{i}=s, A_{i}=a, D_{i}=d\right)$ and $P(a \mid s, 0)=\operatorname{Pr}\left(A_{i}=a \mid S_{i}=s, D_{i}=0\right)$ for any $a, a^{\prime}$ and $c \in \mathcal{A}$. If $a^{\prime}=c$, these bounds are sharp and simplify to the following expression:

$$
\begin{gathered}
\sum_{s \in \mathcal{A}}\left(\lim _{y^{*} \rightarrow-\infty}\left[\int_{y^{*}}^{\infty} 1-\min \left\{1, \frac{F(y \mid s, a, 1)-F(y \mid s, a, 0) P(a \mid s, 0)}{\operatorname{Pr}\left(A_{i}=c \mid S_{i}=s, D_{i}=0\right)}\right\} \mathrm{d} y+y^{*}\right]\right) \operatorname{Pr}\left(S_{i}=s \mid A_{i}=c, D_{i}=0\right) \\
-\mathbb{E}\left[Y_{i} \mid A_{i}=c, D_{i}=0\right] \\
\leq \tau(a, c \mid c) \leq \\
\sum_{s \in \mathcal{A}}\left(\lim _{y^{*} \rightarrow-\infty}\left[\int_{y^{*}}^{\infty} 1-\max \left\{0,1-\frac{1-F(y \mid s, a, 1)+\{1-F(y \mid s, a, 0)\} P(a \mid s, 0)}{\operatorname{Pr}\left(A_{i}=c \mid S_{i}=s, D_{i}=0\right)}\right\} \mathrm{d} y+y^{*}\right]\right) \\
\cdot \operatorname{Pr}\left(S_{i}=s \mid A_{i}=c, D_{i}=0\right)-\mathbb{E}\left[Y_{i} \mid A_{i}=c, D_{i}=0\right]
\end{gathered}
$$

for any $a$ and $c \in \mathcal{A}$.

Intuitively, the observed forced-choice distribution of $Y_{i}(a)$ is a mixture of the choice-specific component distributions, where size of each component is known but the choice-specific distribution is unobserved for all $C_{i} \neq A_{i}$. We bound these unobserved component distributions by first applying the FréchetHoeffding bounds on the joint distribution of $Y_{i}(a)$ and $C_{i}$ for each observed stratum defined by $S_{i}$, conditional on $C_{i} \neq a$. Then, sharp bounds on $\mathbb{E}\left[Y_{i}(a) \mid C_{i}=c\right]$ are derived. A detailed proof can be found in Web Appendix A.3.

We offer several remarks on Proposition 1 . First, the bounds on $\tau\left(a, a^{\prime} \mid c\right)$ are tighter when more units choose the treatment of interest $(c)$ in the free-choice condition. This is because, intuitively, the worstcase assumptions for the unobserved potential outcomes apply to a smaller portion of the population. Second, we can prove the bounds to be sharp only when $a^{\prime}=c$, i.e., when one of the average potential outcomes in $\tau\left(a, a^{\prime} \mid c\right)$ can be point identified from the observed outcome for the free-choice group. This limitation motivates our second set of identification results.

\subsection{Sharp Bounds for Binary Outcomes}

Next, we restrict analysis to outcome variables that are binary $(\mathcal{Y} \in\{0,1\})$ and derive another set of nonparametric bounds on the ACTE. In this case, we can obtain the sharp bounds on $\tau\left(a, a^{\prime} \mid c\right)$ for any $a, a^{\prime}$ and $c \in \mathcal{A}$ (in particular, even when $a \neq a^{\prime} \neq c$ ) by incorporating the full joint distribution of the 
observed variables in the derivation of the bounds. This is achieved via the linear programming approach based on principal stratification (Balke and Pearl, 1997; Frangakis and Rubin, 2002), which has recently been used for nonparametric identification analysis of various causal quantities (e.g. Yamamoto, 2012, Imai et al. 2013). First, we define $2^{J} J^{2}$ principal strata, a partition of the population of units based on the values of their potential outcomes $\left(Y_{i}(0), \ldots, Y_{i}(J-1)\right)$ as well as the values of their stated and revealed preferences $\left(S_{i}\right.$ and $C_{i}$ ). Then we consider the population proportion of each principal stratum, which we denote by $\phi_{y_{0}, \ldots, y_{J-1}, s, c} \equiv \operatorname{Pr}\left(Y_{i}(0)=y_{0}, \ldots, Y_{i}(J-1)=y_{J-1}, S_{i}=s, C_{i}=c\right)$, where $y_{0}, \ldots, y_{J-1} \in\{0,1\}$ and $s, c \in \mathcal{A}$. For the rest of this section, we focus on the case of a tri-valued treatment $(J=3$, as in the media choice example) for notational tractability, although the proposed method can be applied more generally. There are a total of 72 unique principal strata when $J=3$, corresponding to unique combinations in the indices of $\phi_{y_{0}, y_{1}, y_{2}, s, c}$. The proposed method can also be applied to non-binary categorical outcomes with a straightforward extension, which we do not pursue in the current paper in order to keep the exposition simple.

The following proposition shows that the sharp bounds on the ACTE can be obtained by solving a linear programming problem when the outcome is binary.

Proposition 2 (Nonparametric Sharp Bounds on the ACTE for Binary Outcomes) Under Assumptions 1 and 2 and when $J=3$, the nonparametric sharp bounds on $\tau\left(a, a^{\prime} \mid c\right)$ for a binary outcome can be obtained as a solution to the following linear programming problem.

$$
\begin{gathered}
\min _{\Phi} \text { and } \max _{\Phi} \frac{1}{\operatorname{Pr}\left(A_{i}=c \mid D_{i}=0\right)}\left\{\sum_{a^{\prime \prime} \in\{0,1\}} \sum_{s \in \mathcal{A}}\left(\phi_{1,0, y_{a^{\prime \prime}}, s, c}-\phi_{0,1, y_{a^{\prime \prime}}, s, c}\right)\right\}, \\
\text { s.t. } \phi_{y_{0}, y_{1}, y_{2}, s, c^{\prime}} \geq 0 \forall y_{0}, y_{1}, y_{2}, s, c^{\prime}, \sum_{y_{0} \in\{0,1\}} \sum_{y_{1} \in\{0,1\}} \sum_{y_{2} \in\{0,1\}} \sum_{s \in \mathcal{A}} \sum_{c^{\prime} \in \mathcal{A}} \phi_{y_{0}, y_{1}, y_{2}, s, c^{\prime}}=1, \\
\sum_{y_{0} \in\{0,1\}} \sum_{y_{1} \in\{0,1\}} \sum_{y_{2} \in\{0,1\}} \phi_{y_{0}, y_{1}, y_{2}, s, c^{\prime}} \cdot \mathbf{1}\left\{y_{c^{\prime}}=1\right\}=\operatorname{Pr}\left(S_{i}=s, A_{i}=c^{\prime}, Y_{i}=1 \mid D_{i}=0\right) \forall s, c^{\prime}, \\
\sum_{y_{0} \in\{0,1\}} \sum_{y_{1} \in\{0,1\}} \sum_{y_{2} \in\{0,1\}} \phi_{y_{0}, y_{1}, y_{2}, s, c^{\prime}}=\operatorname{Pr}\left(S_{i}=s, A_{i}=c^{\prime} \mid D_{i}=0\right) \forall s, c^{\prime}, \text { and } \\
\sum_{y_{0} \in\{0,1\}} \sum_{y_{1} \in\{0,1\}} \sum_{y_{2} \in\{0,1\}} \sum_{c^{\prime} \in \mathcal{A}} \phi_{y_{0}, y_{1}, y_{2}, s, c^{\prime}} \cdot \mathbf{1}\left\{y_{a^{\prime \prime}}=1\right\}=\operatorname{Pr}\left(S_{i}=s, Y_{i}=1 \mid A_{i}=a^{\prime \prime}, D_{i}=\right. \\
\text { 1) } \forall s, a^{\prime \prime}, \text { where } \Phi \equiv\left\{\phi_{y_{0}, y_{1}, y_{2}, s, c}: y_{0} \in\{0,1\}, y_{1} \in\{0,1\}, y_{2} \in\{0,1\}, s \in \mathcal{A}, c \in \mathcal{A}\right\} .
\end{gathered}
$$

A proof is provided in Web Appendix A.4. The maximization and minimization problems in Proposi- 
tion 2 are standard linear programming problems which can be easily solved numerically with given data using statistical software, such as the lpSolve package in R. We note that, for the $\tau(a, c \mid c)$ case with binary outcomes, equation (7) simplifies to

$$
\begin{aligned}
& \sum_{s \in \mathcal{A}}\left(1-\min \left\{1, \frac{F(0 \mid s, a, 1)-F(0 \mid s, a, 0) P(a \mid s, 0)}{\operatorname{Pr}\left(A_{i}=c \mid S_{i}=s, D_{i}=0\right)}\right\}\right) \operatorname{Pr}\left(S_{i}=s \mid A_{i}=c, D_{i}=0\right) \\
& \leq \tau(a, c \mid c) \leq \\
& -\operatorname{Pr}\left(Y_{i}=1 \mid A_{i}=c, D_{i}=0\right) \\
& \sum_{s \in \mathcal{A}}\left(1-\max \left\{0,1-\frac{1-F(0 \mid s, a, 1)+\{1-F(0 \mid s, a, 0)\} P(a \mid s, 0)}{\operatorname{Pr}\left(A_{i}=c \mid S_{i}=s, D_{i}=0\right)}\right\}\right) \operatorname{Pr}\left(S_{i}=s \mid A_{i}=c, D_{i}=0\right) \\
& -\operatorname{Pr}\left(Y_{i}=1 \mid A_{i}=c, D_{i}=0\right),
\end{aligned}
$$

which we find to numerically coincide with the linear programming bounds based on Proposition 2 , as they should.

\section{Sensitivity Analysis}

The nonparametric bounds in Propositions 1 and 2 represent "worst-case" scenarios, in that they allow for the maximal deviation in the average potential outcomes between those subjects who merely state they would take a treatment and those who actually choose to take the treatment. In contrast, the naïve estimator given in equation (5) relies on Assumption 3 and assumes (often demonstrably falsely) that this deviation is zero. The truth, however, lies somewhere between these two extremes.

In this section, we propose a sensitivity analysis to investigate this middle ground. Sensitivity analysis is a commonly used inferential strategy where the degree of violation of a key identification assumption is quantified via a sensitivity parameter (Rosenbaum, 2002) and the consequence of this violation is then expressed and analyzed as a function of this parameter. Here, we consider a sensitivity parameter $\rho_{a c}$ which represents the maximum absolute difference we allow to exist between the average of a potential outcome $\left(Y_{i}(a)\right)$ among those who state a particular treatment preference $\left(S_{i}=c\right)$ and the average of the same potential outcome among those who actually choose that treatment $\left(C_{i}=c\right)$. Formally, $\rho_{a c}$ is 
defined by the following inequality,

$$
\left|\mathbb{E}\left[Y_{i}(a) \mid S_{i}=c\right]-\mathbb{E}\left[Y_{i}(a) \mid C_{i}=c\right]\right| \leq \rho_{a c}
$$

which implies the following additional constraint for identification analysis under Assumptions 1 and 2 ,

$$
\mathbb{E}\left[Y_{i} \mid S_{i}=c, A_{i}=a, D_{i}=1\right]-\rho_{a c} \leq \mathbb{E}\left[Y_{i}(a) \mid C_{i}=c\right] \leq \mathbb{E}\left[Y_{i} \mid S_{i}=c, A_{i}=a, D_{i}=1\right]+\rho_{a c}
$$

for a given pair of $a$ and $c \in \mathcal{A}$ such that $a \neq c$.

The proposed sensitivity analysis proceeds by combining the sensitivity constraint in equation (8) with the no-assumption bounds on average choice-specific potential outcomes,

$\sum_{s \in \mathcal{A}}\left\{\pi^{-}(a \mid s, c) \operatorname{Pr}\left(S_{i}=s \mid A_{i}=c, D_{i}=0\right)\right\} \leq \mathbb{E}\left[Y_{i}(a) \mid C_{i}=c\right] \leq \sum_{s \in \mathcal{A}}\left\{\pi^{+}(a \mid s, c) \operatorname{Pr}\left(S_{i}=s \mid A_{i}=c, D_{i}=0\right)\right\}$ where $\pi^{-}(a \mid s, c)$ and $\pi^{+}(a \mid s, c)$ are the sharp bounds on $\mathbb{E}\left[Y_{i}(a) \mid S_{i}=s, C_{i}=c\right]$ derived in Web Appendix A.3. We therefore find bounds on $\tau\left(a, a^{\prime} \mid c\right)$ for a given pair of $\left(\rho_{a c}, \rho_{a^{\prime} c}\right)$ by the interval difference

$$
\begin{aligned}
\tau\left(a, a^{\prime} \mid c\right) \in\left(\left[\mathbb{E}\left[Y_{i} \mid S_{i}=c, A_{i}=a, D_{i}=1\right]-\rho_{a c}, \mathbb{E}\left[Y_{i} \mid S_{i}=c, A_{i}=a, D_{i}=1\right]+\rho_{a c}\right]\right. \\
\left.\bigcap\left[\sum_{s \in \mathcal{A}}\left\{\pi^{-}(a \mid s, c) \operatorname{Pr}\left(S_{i}=s \mid A_{i}=c, D_{i}=0\right)\right\}, \sum_{s \in \mathcal{A}}\left\{\pi^{+}(a \mid s, c) \operatorname{Pr}\left(S_{i}=s \mid A_{i}=c, D_{i}=0\right)\right\}\right]\right) \\
-\left(\left[\mathbb{E}\left[Y_{i} \mid S_{i}=c, A_{i}=a^{\prime}, D_{i}=1\right]-\rho_{a^{\prime} c}, \mathbb{E}\left[Y_{i} \mid S_{i}=c, A_{i}=a^{\prime}, D_{i}=1\right]+\rho_{a c}\right]\right. \\
\left.\bigcap\left[\sum_{s \in \mathcal{A}}\left\{\pi^{-}\left(a^{\prime} \mid s, c\right) \operatorname{Pr}\left(S_{i}=s \mid A_{i}=c, D_{i}=0\right)\right\}, \sum_{s \in \mathcal{A}}\left\{\pi^{+}\left(a^{\prime} \mid s, c\right) \operatorname{Pr}\left(S_{i}=s \mid A_{i}=c, D_{i}=0\right)\right\}\right]\right) .
\end{aligned}
$$

Again, when $a^{\prime}=c$, this expression can be simplified by substituting the second interval with the observed quantity $\mathbb{E}\left[Y_{i} \mid A_{i}=c, D_{i}=0\right]$, and the resulting bounds are now sharp for a given value of $\rho_{a c}$. When $a^{\prime} \neq c$, we recommend setting $\rho_{a c}=\rho_{a^{\prime} c}=\rho$ and conducting the analysis with respect to a single sensitivity parameter for both counterfactual outcomes for the sake of interpretability.

Before proceeding to binary outcomes, we note that a similar sensitivity approach can be applied to a simpler design with only the forced-exposure arm. However, this approach is limited in two ways. 
First, the sensitivity bounds will be much wider because they will consist of Equation 9 without the latter portion of each intersection. Second, because the free-choice arm is the only source of information about the feasible range of $\rho_{a c}$, even infinite values cannot be excluded in the simpler design when the outcome variable is unbounded. At this extreme, the sensitivity ananalysis becomes completely uninformative, as noted in Section 4

For binary outcomes, the sharp sensitivity bounds can be numerically obtained for any $\tau\left(a, a^{\prime} \mid c\right)$ and given values of $\rho_{a c}$ and $\rho_{a^{\prime} c}$ by incorporating equation (8) into the linear programming problem in Proposition 2 as another set of linear constraints. For the special case of $J=3$, these constraints can be written in terms of $\phi_{y_{0}, y_{1}, y_{2}, s, c}$ as $\sum_{y_{0} \in\{0,1\}} \sum_{y_{1} \in\{0,1\}} \sum_{y_{2} \in\{0,1\}} \sum_{s \in \mathcal{A}} \phi_{y_{0}, y_{1}, y_{2}, s, c} \mathbf{1}\left\{y_{a^{*}}=1\right\} \geq$ $\left\{\operatorname{Pr}\left(Y_{i}=1 \mid S_{i}=c, A_{i}=a^{*}, D_{i}=1\right)-\rho_{a^{*} c}\right\} \operatorname{Pr}\left(A_{i}=c \mid D_{i}=0\right)$ and $\sum_{y_{0} \in\{0,1\}} \sum_{y_{1} \in\{0,1\}} \sum_{y_{2} \in\{0,1\}}$ $\sum_{s \in \mathcal{A}} \phi_{y_{0}, y_{1}, y_{2}, s, c} \mathbf{1}\left\{y_{a^{*}}=1\right\} \leq\left\{\operatorname{Pr}\left(Y_{i}=1 \mid S_{i}=c, A_{i}=a^{*}, D_{i}=1\right)+\rho_{a^{*} c}\right\} \operatorname{Pr}\left(A_{i}=c \mid D_{i}=0\right)$ for given $c$ and $a^{*} \in\left\{a, a^{\prime}\right\}$.

\section{Statistical Inference}

In this section, we discuss our methods for performing statistical inference for the large-sample bounds in Sections 4 and 5. Several approaches have been proposed for inference about partially identified parameters in the literature, including the nonparametric bootstrap (e.g. Horowitz and Manski, 2000). Here, we take a Bayesian approach where we obtain simulated draws from the marginal posterior distribution for the bounds on $\tau\left(a, a^{\prime} \mid c\right)$ by Monte Carlo integration of the approximated joint posterior.

For inference about the general bounds in Proposition 1, we use the following procedure to obtain one simulated draw of the bounds, $\tau^{-}\left(a, a^{\prime} \mid c\right)^{*}$ and $\tau^{+}\left(a, a^{\prime} \mid c\right)^{*}$, from their joint posterior.

\section{Algorithm 1 (Posterior Simulation for the Bounds in Proposition 1)}

1. Draw $\boldsymbol{p} \equiv\left[p_{s}\right] \sim \operatorname{Dirichlet}(\boldsymbol{n})$, where $\boldsymbol{n} \equiv\left[n_{s}\right]=\left[\sum_{i=1}^{N} \mathbf{1}\left\{S_{i}=0\right\}, \cdots, \sum_{i=1}^{N} \mathbf{1}\left\{S_{i}=J-1\right\}\right]^{\top}$.

2. For each $s \in \mathcal{A}$ :

(a) Draw $\boldsymbol{q}_{s} \equiv\left[q_{s a}\right] \sim \operatorname{Dirichlet}\left(\boldsymbol{n}_{s}^{0}\right)$, where $\boldsymbol{n}_{s}^{0} \equiv\left[n_{s a}^{0}\right]=\left[\sum_{i=1}^{N} \mathbf{1}\left\{S_{i}=s, A_{i}=0, D_{i}=\right.\right.$ $\left.0\}, \cdots, \sum_{i=1}^{N} \mathbf{1}\left\{S_{i}=s, A_{i}=J-1, D_{i}=0\right\}\right]^{\top} ;$ 
(b) For each a and $c \in \mathcal{A}$, draw a pair $\left[\pi^{-}(a \mid s, c), \pi^{+}(a \mid s, c)\right]$ from Normal $\left(\left[\begin{array}{l}\bar{\pi}^{-} \\ \bar{\pi}^{+}\end{array}\right],\left[\begin{array}{cc}V_{C}^{-} & C \\ C & V^{+}\end{array}\right]\right)$, where expressions for $\bar{\pi}^{-}, \bar{\pi}^{+}, V^{-}, V^{+}$and $C$ are provided in Web Appendix A.5.

3. Calculate a simulated draw of $\left[\tau^{-}\left(a, a^{\prime} \mid c\right), \tau^{+}\left(a, a^{\prime} \mid c\right)\right]$ as

$$
\begin{aligned}
\tau^{-}\left(a, a^{\prime} \mid c\right)^{*} & =\sum_{s \in \mathcal{A}}\left(\pi^{-}(a \mid s, c)-\pi^{+}\left(a^{\prime} \mid s, c\right)\right) \frac{q_{s c} p_{s}}{\sum_{s^{\prime} \in \mathcal{A}} q_{s^{\prime} c} p_{s^{\prime}}} \\
\tau^{+}\left(a, a^{\prime} \mid c\right)^{*} & =\sum_{s \in \mathcal{A}}\left(\pi^{+}(a \mid s, c)-\pi^{-}\left(a^{\prime} \mid s, c\right)\right) \frac{q_{s c} p_{s}}{\sum_{s^{\prime} \in \mathcal{A}} q_{s^{\prime} c} p_{s^{\prime}}} .
\end{aligned}
$$

Note that for $a=c, \pi(a \mid s, c)=\pi^{-}(a \mid s, c)=\pi^{+}(a \mid s, c)$ and Step 2b will be a draw from the univariate normal distribution with mean $\bar{\pi}$ and variance $V$, which are also given in Web Appendix A.5. This procedure asymptotically approximates the posterior for the bounds on $\tau\left(a, a^{\prime} \mid c\right)$ without assuming a full parametric model for the true distribution of the potential outcomes. Exact prior specifications are therefore unnecessary for these parameters; We use improper flat priors where explicit prior specifications are necessary (i.e., for $\boldsymbol{p}$ and $\boldsymbol{q}$ ). Details are provided in Web Appendix A.5.

For the sharp bounds for binary outcomes in Proposition 2, we can apply a similar algorithm which simulates the joint posterior on the proportions of the observed strata and solves the linear programming problem using the simulated draws from the posterior. The procedure is identical to Algorithm 1 except that Steps $2 \mathrm{~b}$ and 3 should be replaced with the following:

2. (b) For each $a \in \mathcal{A}$, $\operatorname{draw} H_{s a} \sim \operatorname{Beta}\left(\sum_{i=1}^{N} \mathbf{1}\left\{Y_{i}=1, S_{i}=s, A_{i}=a, D_{i}=1\right\}, \sum_{i=1}^{N} \mathbf{1}\left\{Y_{i}=\right.\right.$ $\left.\left.0, S_{i}=s, A_{i}=a, D_{i}=1\right\}\right)$ and $G_{s a} \sim \operatorname{Beta}\left(\sum_{i=1}^{N} 1\left\{Y_{i}=1, S_{i}=s, A_{i}=a, D_{i}=\right.\right.$ $\left.0\}, \sum_{i=1}^{N} \mathbf{1}\left\{Y_{i}=0, S_{i}=s, A_{i}=a, D_{i}=0\right\}\right)$.

3. Calculate a simulated draw of $\left[\tau^{-}\left(a, a^{\prime} \mid c\right), \tau^{+}\left(a, a^{\prime} \mid c\right)\right]$ by solving the linear programming problem in Proposition 2, with the probabilities of the observed strata in the constraints replaced with the simulated draws from their posterior $\left(\boldsymbol{q} \equiv\left[\boldsymbol{q}_{s}\right], \boldsymbol{G} \equiv\left[G_{s a}\right]\right.$ and $\left.\boldsymbol{H} \equiv\left[H_{s a}\right]\right)$.

Once we generate a large enough number of draws from the approximated joint posterior of $\tau^{-}\left(a, a^{\prime} \mid c\right)$ and $\tau^{+}\left(a, a^{\prime} \mid c\right)$, we construct $100(1-\alpha) \%$ credible intervals for the bounds. For each combination of $a$, 
$a^{\prime}$ and $c$, we use a numerical procedure to find the narrowest interval that entirely contains $1-\alpha$ of the draws of the bounds (i.e., $\left[\hat{\tau}^{-}\left(a, a^{\prime} \mid c\right), \hat{\tau}^{+}\left(a, a^{\prime} \mid c\right)\right]$ such that $\hat{\tau}^{-}\left(a, a^{\prime} \mid c\right)<\tau^{-}\left(a, a^{\prime} \mid c\right)^{*}$ and $\tau^{+}\left(a, a^{\prime} \mid c\right)^{*}<$ $\hat{\tau}^{+}\left(a, a^{\prime} \mid c\right)$ for $100(1-\alpha) \%$ of the draws). We call these the highest posterior density (HPD) intervals for the bounds, because they contain $1-\alpha$ of the posterior probability mass defined on the bounds with the minimal width. In practice, we find that HPD intervals are virtually indistinguishable from simply taking the $\alpha / 2$ and $1-\alpha / 2$ quantiles of the lower and upper bounds posteriors, respectively. In a simulation study (Web Appendix A.7), we find that HPD intervals generally perform better than a nonparametric bootstrap approach in finite samples in terms of frequentist coverage probability.

Finally, uncertainty estimates for the sensitivity analysis bounds in Section 5 can also be obtained following analogous procedures. For the general bounds, the key difference is that the algorithms for the sensitivity bounds are augmented to incorporate an additional set of parameters $\mathbb{E}\left[Y_{i} \mid S_{i}, A_{i}, D_{i}=1\right]$ as part of the joint posterior to simulate from, which is used in the sensitivity constraint. Details are provided in Web Appendix A.6.

\section{Empirical Application}

Now we apply the proposed methodology to the empirical example we described in Section 2 .

\subsection{Design and Data}

In implementing the media choice experiment, we closely followed the proposed protocol as described in Section 3.1 and summarized in Figure 1. First, to measure the stated preferences over treatment options, early in the survey we asked, "If you were given the choice of the following four television programs to watch, which would you choose?" We presented each television choice (listed in Section 2) with an accompanying screenshot of the host of the show, while randomizing the order of the shows.

Subsequently, we included a "washout" period in which we asked subjects various questions not directly related to the media choice (e.g. demographics, unrelated psychological experiments). Survey researchers have long known that the order in which questions are asked can influence subsequent 
patterns of responses. One reason is that asking a respondent a particular question can prime certain concepts, making them salient at the time of response (Tourangeau et al., 2000). In this case, we included these filler questions to minimize the possibility that responses to the initial preference question might contaminate their subsequent media choice in the free-choice condition.

The washout items included questions about their partisanship that we used to categorize their media preferences as pro- or counter-attitudinal. We considered Fox News "pro-attitudinal" for Republicans and "counter-attitudinal" for Democrats. MSNBC was coded in the opposite manner. After excluding subjects who were neither Democrats nor Republicans, $31 \%$ of the sample expressed a preference for pro-attitudinal media $\left(S_{i}=0\right), 12 \%$ for counter-attitudinal media $\left(S_{i}=1\right)$, and the remaining $56 \%$ for an entertainment show $\left(S_{i}=2\right)$.

Next, we randomized subjects with equal probability into the forced-exposure $\left(D_{i}=1\right)$ and freechoice $\left(D_{i}=0\right)$ conditions. We randomly assigned those in the forced-choice arm to watch proattitudinal media $\left(A_{i}=0\right)$, counter-attitudinal media $\left(A_{i}=1\right)$, or a randomly chosen entertainment program $\left(A_{i}=2\right)$, each with probability $1 / 3$. For those in the free-choice arm, we instead asked, "Which of these programs would you like to watch now?" with the same four options presented as before. Based on their partisanship and response, we recorded the actual choice $C_{i}$ as 0,1 , or 2 . Here, we find that stated preferences correspond only loosely to actual choices, and that those stating a preference for entertainment were more likely to be consistent in their actual choices $\left(\operatorname{Pr}\left(C_{i}=2 \mid S_{i}=2\right)=0.91\right.$, whereas $\operatorname{Pr}\left(C_{i}=0 \mid S_{i}=0\right)=0.83$ and $\operatorname{Pr}\left(C_{i}=1 \mid S_{i}=1\right)=0.78$ in the data). We assigned these subjects to view their choice, so that $A_{i}=C_{i}$ in the free-choice arm.

We consider two outcome variables. First, after showing the program, we asked respondents to rate the clip they watched on a number of dimensions, which we subsequently summarized into an index of sentiment toward media. The index ranged between 0 and 1 and the mean and standard deviation were 0.61 and 0.17 , respectively. Second, to gauge behavioral responses, we asked subjects how likely they would be to discuss the clip with a friend, which we recoded into a binary indicator. Overall, $62.5 \%$ of subjects were at least "somewhat likely" to discuss the viewed program $(=1)$ while the rest answered 
Free-choice Condition $\left(D_{i}=0\right)$

\begin{tabular}{|c|c|c|c|c|c|c|c|c|c|c|}
\hline \multirow{2}{*}{\multicolumn{2}{|c|}{$\begin{array}{l}\text { Stated Preference }\left(S_{i}\right) \\
\text { Actual Choice }\left(C_{i}=A_{i}\right)\end{array}$}} & \multicolumn{3}{|c|}{0} & \multicolumn{3}{|c|}{1} & \multicolumn{3}{|c|}{2} \\
\hline & & 0 & 1 & 2 & 0 & 1 & 2 & 0 & 1 & 2 \\
\hline Strata proportic & & .25 & .02 & .03 & .01 & .09 & .02 & .03 & .02 & .53 \\
\hline \multirow{2}{*}{ Outcomes $\left(Y_{i}\right)$} & Sentir & .67 & .51 & .66 & .52 & .56 & .60 & .60 & .54 & .68 \\
\hline & Likely to discuss & .77 & .76 & .62 & .62 & .75 & .68 & .82 & .77 & .57 \\
\hline
\end{tabular}

Forced-exposure Condition $\left(D_{i}=1\right)$

\begin{tabular}{|c|c|c|c|c|c|c|c|c|c|c|}
\hline Stated Preferen & $\mathrm{e}\left(S_{i}\right)$ & \multicolumn{3}{|c|}{0} & \multicolumn{3}{|c|}{1} & \multicolumn{3}{|c|}{2} \\
\hline Randomized Tr & atment $\left(A_{i}\right)$ & 0 & 1 & 2 & 0 & 1 & 2 & 0 & 1 & 2 \\
\hline Strata proportic & & .10 & .11 & .11 & .04 & .05 & .05 & .20 & .18 & .16 \\
\hline & Sen & .67 & .38 & .64 & .59 & .54 & .63 & .57 & .47 & .64 \\
\hline Outcomes ( $Y$ & Likely to discuss & .74 & .48 & .42 & .73 & .76 & .66 & .66 & .56 & .56 \\
\hline
\end{tabular}

Table 1: Summary of Observed Data in the Media Choice Experiment. The third row in each table shows the observed proportion in each stated preference-treatment stratum. The bottom two rows in each table represent the sample averages of the two outcome variables in each stratum.

they were "not likely" to do so $(=0)$.

Table 1 summarizes the observed data from the media choice experiment. The general pattern indicates that discrepancies between stated and true preferences not only exist, but that these discrepancies are also associated with different responses to media. For example, among those respondents in the freechoice group who stated a preference for pro-attitudinal media and also chose a pro-attitudinal program, mean sentiment was 0.67 . In contrast, responses were significantly lower (by 0.07) among free-choice subjects who stated a preference for entertainment but actually chose pro-attitudinal media.

\subsection{Nonparametric Bounds}

Given the evidence that stated preferences of subjects do not accurately reflect their actual choices, we now seek to bound the ACTEs using the method developed in Section 4 . Figure 2 presents the resulting nonparametric bounds, along with their $95 \%$ posterior intervals obtained via the procedure proposed in Section 6, The left panel presents results for subjects' sentiment toward the media watched (Proposition 11, and the right panel presents results for whether respondents were likely to discuss the story with a friend (binary; Proposition 2). Each vertically arrayed plot depicts the effect of a particular change in the assigned media, from entertainment to pro-attitudinal (top), entertainment to counter-attitudinal (mid- 
Sentiment toward Media

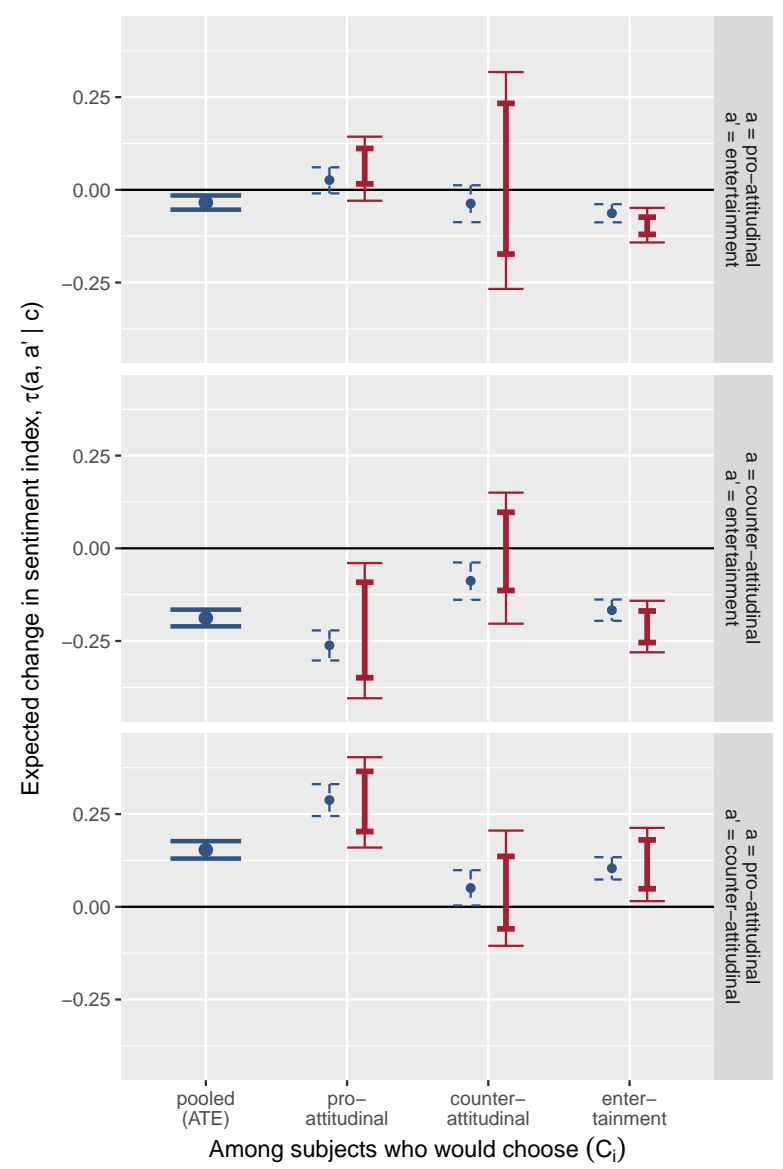

Discuss Story with Friends (binary)

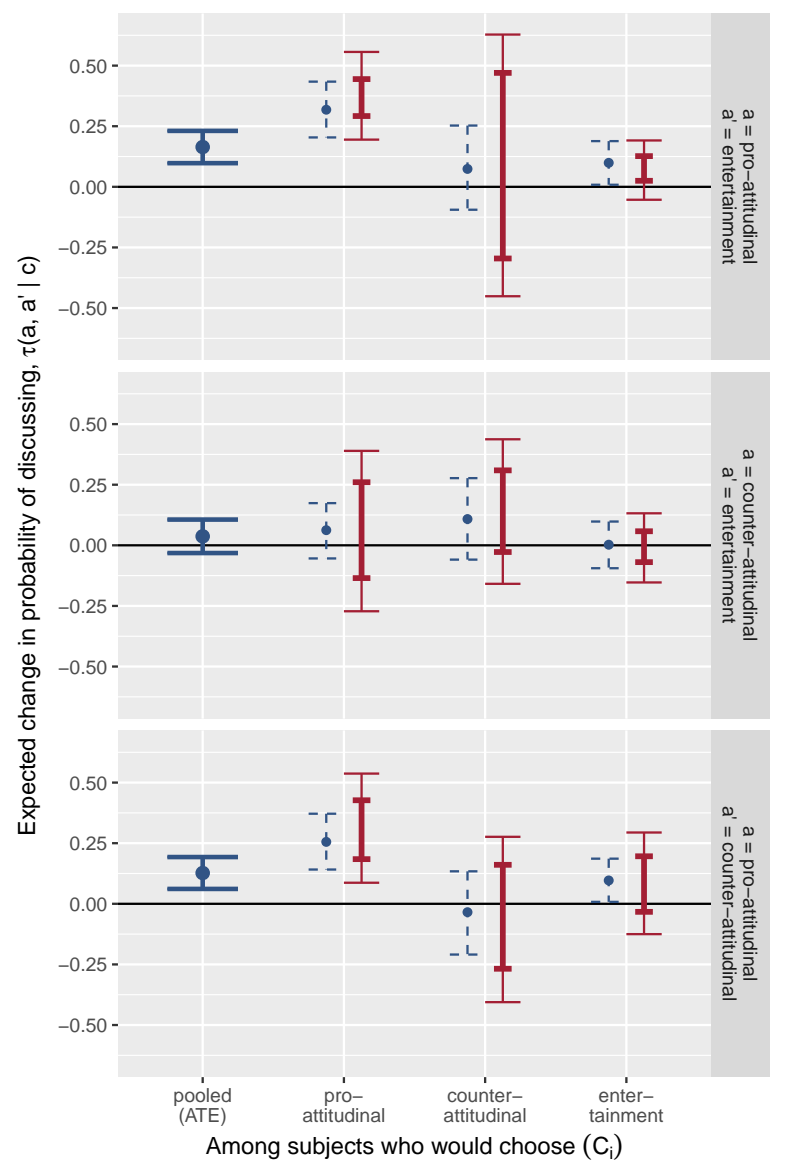

Figure 2: Estimated Nonparametric Bounds on the ACTE of Partisan News Media. Vertically stacked plots correspond to the same outcome variable. Horizontally aligned plots depict the effect of a particular change in the assigned media, i.e., $\mathbb{E}\left[Y_{i}(a)-Y_{i}\left(a^{\prime}\right) \mid C_{i}=c\right]$. Pairs of lines correspond to the ACTE among those that would choose a given media (horizontal axis labels). Large blue points and solid thick blue error bars are pooled ATEs. Small blue points are naïve estimates, with blue dashed error bars representing $95 \%$ asymptotic confidence intervals. Solid thick red error bars are estimated bounds (posterior means) and thin error bars give $95 \%$ posterior intervals.

dle) and counter- to pro-attitudinal (bottom). The leftmost blue solid circle (point estimate) and error bar (95\% asymptotic confidence interval) in each plot is the pooled ATE. Paired lines within each plot (thin blue and thick red) represent the estimated ACTE of that treatment among subjects that would choose pro-attitudinal media (left), counter-attitudinal media (middle) and an entertainment show (right). Small blue points are the point estimates under Assumptions 1, 2 and 3, i.e., the naïve estimates that assume the ignorability of the discrepancy between stated preferences and actual choices. Blue dashed error bars are 95\% asymptotic confidence intervals. Solid red error bars are nonparametric bounds on ACTEs under 
Assumptions 1 and 2 alone, with thick lines representing estimated bounds (posterior means) and thin lines representing posterior intervals.

For example, consider the middle bars in the center left plot. Here, blue dashed estimates show that, even among subjects that state a preference for counter-attitudinal media, this media results in more negative sentiment than entertainment—while small, the naïve estimate is negative and statistically significant at the $95 \%$ confidence level. In contrast, the sharp bounds, centered directly on zero, show that this result may be misleading for the group that would actually choose counter-attitudinal media, because inconsistency in stated and true preferences may be systematically correlated with responses. Indeed, in Section 7.3 , we will show that it is highly sensitive to assumptions about the informativeness of the stated preference. The greatest source of this discrepancy is that for counter-attitudinal media, stated preferences are particularly inconsistent with actual choices. In the free-choice condition, over $20 \%$ of subjects stating this preference went on to choose other media.

We now briefly discuss the remaining estimates in the left panel of Figure 2, starting with the top left and proceeding clockwise. In the top plot, all bounds agree with naïve estimates: Differences in sentiment toward pro-attitudinal media and entertainment are indistinguishable, except for a small adverse reaction among those with a true preference for entertainment (top right). These same subjects have a significant and seemingly larger adverse reaction to counter-attitudinal media (center right), and the difference between pro- and counter-attitudinal media among this group is statistically significant (lower right). Among units that would choose counter-attitudinal media, naïve estimates suggest a significantly more positive reaction to pro- versus counter-attitudinal media (lower middle), but these results again implicitly rest on strong assumptions about the informativeness of stated preferences. Not surprisingly, those who would choose pro-attitudinal media react more positively toward it than toward counter-attitudinal media (lower left). Finally, estimated bounds appear to support the naïve estimate that those who would choose pro-attitudinal media have a negative response to counter-attitudinal media (versus entertainment, center left) and these bounds are statistically distinct from zero.

Finally, we present nonparametric sharp bounds for the binary outcome of whether subjects are likely 
to discuss the story with a friend. As explained in Section 4.2, these are the narrowest possible bounds that can be found with the available information. We discuss statistically significant results only. Among units that would choose pro-attitudinal media, bounds validate the naïve estimate that this media has a large effect on the dissemination of information, both relative to entertainment (top left) and relative to counter-attitudinal media (bottom left). Naïve estimates suggest a similar but smaller pattern of effects for those who would choose entertainment. However, the estimated bounds are respectively consistent with the naïve estimate in sign but statistically inconclusive (versus entertainment, top right) and entirely inconclusive (versus counter-attitudinal media, bottom right).

\subsection{Sensitivity Analysis}

Next, we apply the sensitivity analysis developed in Section 5 and show how the bounds become tighter as we allow less difference between the average potential outcomes conditional on a stated preference versus actual choice $(\rho)$. For illustration, we focus on the sentiment index. The results are presented in Figure 3. The results for the binary discussion indicator are in Figure A.1 in the Web Appendix.

Using bounds on mean choice-specific potential outcomes (not presented), we find that the estimated maximal difference for any strata is 0.18 , approximately one standard deviation in the outcome variable. Thus, in Figure 3, estimated sensitivity results have converged to the estimated bounds at or below this level of $\rho$. For most strata, differences above 0.1 can be ruled out. For some ACTEs, sensitivity results are not shown for low values of $\rho$ because in this region, it becomes impossible to simultaneously satisfy the sensitivity constraints implied by $\rho$ and the naïve results, on the one hand, and the bounding constraints, on the other.

For illustration we focus on the middle plot in the center row of Figure 3 . Neglecting sampling error, the true value of $\rho$ here should lie somewhere in $[0.02,0.18]$. The naïve estimates suggest that counterattitudinal media negatively affects media sentiment (relative to entertainment) even among those who would choose counter-attitudinal media (middle plot), somewhat surprisingly. However, the upper bound is statistically indistinguishable from zero when Assumption 3 is even slightly relaxed by $\rho=0.03$. 
Sensitivity Analysis for Sentiment toward Media

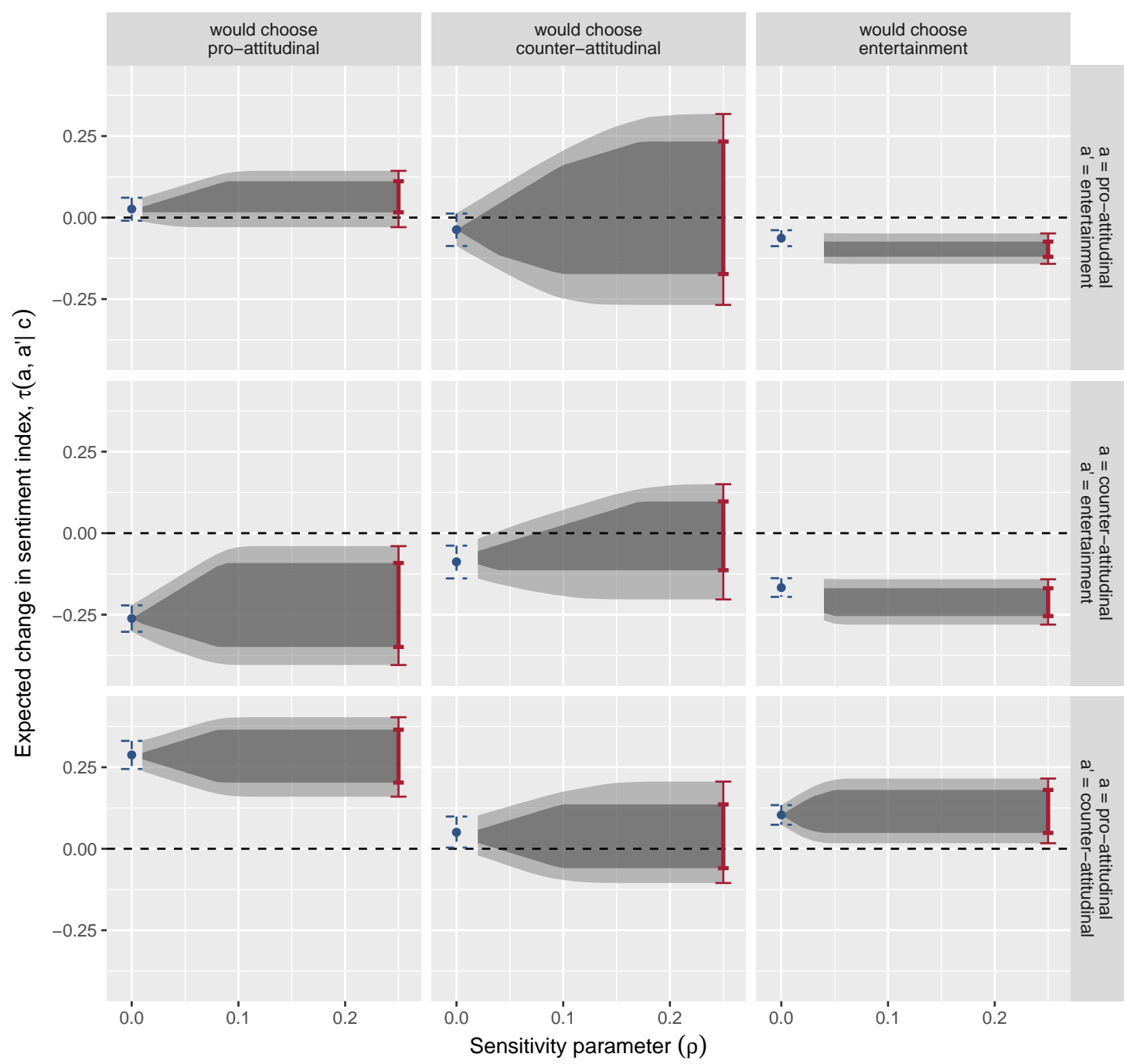

Figure 3: Sensitivity Analysis for the ACTE of Partisan News Media. The plots correspond to the left panel of Figure 2. On the left side of each plot, a blue point and error bars represent the naïve estimate and $95 \%$ asymptotic confidence intervals, respectively. On the right side, thick red error bars represent no-assumption bounds and thin red error bars represent $95 \%$ posterior intervals. The dark shaded region between these depicts how bounds grow narrower as additional information from the naïve estimates are incorporated ( $\rho_{a c}=\rho_{a^{\prime} c}=\rho$ grows small). Lightly shaded regions are $95 \%$ posterior regions for sensitivity results.

Estimated bounds include zero for values of $\rho>0.07$, less than half of a standard deviation in the observed outcome variable. 


\section{Simulations}

To evaluate the finite-sample performance of our procedure in a naturalistic setting, we conduct MonteCarlo simulations that are based closely on the observed data from our empirical application. We design our simulation study to consider two challenges that regularly arise in PPTs: the discrepancy between stated preferences and actual choices, and the possibility that choices are related to potential outcomes in an unobservable way. We quantify these complications via a "choice divergence" (CD) parameter and an "outcome divergence" (OD) parameter, respectively, and examine how bias and coverage rates are affected as they become more severe.

\subsection{Divergence of actual choices from stated preferences}

In our first set of simulations, we begin by generating a hypothetical population for which the observable margins are identical to those of our empirical sample. For the unobserved variables, such as $Y_{i}(a)$ for $C_{i} \neq a$, we make the most generous assumption possible - that potential outcomes among the unobserved choice-based subgroups are identical in distribution — and generate the values accordingly. This forms our baseline simulation data, in which Assumption 3 is satisfied and therefore the naïve estimator for the ACTE should perform well.

We then introduce the CD parameter, which takes on values in $[0,1]$ and captures the informativeness of the stated preferences. That is, we regenerate the actual choice $C_{i}^{*}$ such that $\operatorname{Pr}\left(C_{i}^{*}=c \mid S_{i}=s\right)=$ $(1-\mathrm{CD}) \operatorname{Pr}\left(C_{i}=c \mid S_{i}=s\right)+\mathrm{CD} / J$, where $C_{i} \in\{1, \ldots, J\}$ for each $c$ and $s$. When $\mathrm{CD}=0$, the joint distribution of $C_{i}^{*}$ and $S_{i}$ is identical to that of the experimental sample (i.e., with $87 \%$ overall agreement between stated preferences and actual choices). When $\mathrm{CD}=1$, stated preferences are entirely uninformative, and the choice probabilities are $1 / 3$ for every treatment.

For each value at which the CD parameter is set $(0,1 / 3,2 / 3$ and 1$)$, we draw 500 sample datasets of size 3,000. We focus on $\tau(0,2 \mid 0)$ for illustration. Naïve ACTE estimates and bounds estimates are computed in each sample dataset, averaged, and compared to the population ACTE and bounds to assess 


\begin{tabular}{rrrrr}
\hline & $\mathrm{CD}=0.00$ & $\mathrm{CD}=0.33$ & $\mathrm{CD}=0.67$ & $\mathrm{CD}=1.00$ \\
\hline naïve & 0.002 & 0.011 & 0.023 & 0.038 \\
$\min$ & -0.001 & -0.001 & -0.001 & 0.000 \\
$\max$ & -0.001 & -0.001 & -0.001 & -0.001 \\
\hline
\end{tabular}

\begin{tabular}{rrrrr}
\hline $\mathrm{N}$ & $\mathrm{CD}=0.00$ & $\mathrm{CD}=0.33$ & $\mathrm{CD}=0.67$ & $\mathrm{CD}=1.00$ \\
\hline 500 & 0.959 & 0.944 & 0.914 & 0.926 \\
1000 & 0.956 & 0.926 & 0.930 & 0.924 \\
3000 & 0.946 & 0.936 & 0.916 & 0.946 \\
10000 & 0.938 & 0.938 & 0.934 & 0.930 \\
50000 & 0.946 & 0.944 & 0.940 & 0.948 \\
\hline
\end{tabular}

Table 2: Estimated Bias and Coverage Rates for Various CD Values. Results for each CD value are based on 500 sample datasets. The bias results for the naïve and the bounds lower/upper endpoint estimators (left) are based on datasets of size $N=3000$. The bounds posterior interval coverage rates (right) have Monte Carlo standard errors of approximately 0.01 .

bias. The results of these simulations are collected in Table 2 (left panel) and show that the bias of the naïve estimator increases as stated preferences diverge more from actual choices. At the extreme, the bias of the naïve estimate is well over double the standard error of the naïve estimate in a particular sample dataset (roughly 0.016 at this sample size). In contrast, ACTE bounds estimates are centered almost exactly on their population analogue, and bias is unaffected by the CD parameter.

For each sample dataset, we also implement the procedure described in Section 6 to compute $95 \%$ posterior intervals on the bounds. The proportion of intervals that fully contain the population bounds is reported in Table 2 (right panel) for sample sizes of ranging from 500 to 50,000 units. We find that when stated preferences are highly divergent from actual choices (CD is high), coverage rates are less than nominal with small sample sizes, but they become indistingishable from $95 \%$ as the number of observations increase. We note that when the bounds are wide relative to their credible interval, coverage of the population bounds at level $1-\alpha$ corresponds to a worst-case coverage of the population ACTE at level $1-\alpha / 2$. In Web Appendix A.7, the proposed method is shown to outperform both alternative bootstrap-based approach and an extension to the parametric model of Long et al. (2008).

\subsection{Divergence of outcomes between choice groups}

Next, we define the outcome divergence (OD) parameter, also in $[0,1]$, which controls the distributions of the unobservable potential outcomes, $\operatorname{Pr}\left(Y_{i}(a) \leq y \mid S_{i}=s, C_{i}=c, D_{i}=0\right)$ when $c \neq a$. Specifically, we generate missing potential outcomes for the free-choice group such that $\operatorname{Pr}\left(Y_{i}(a) \leq\right.$ $\left.y \mid S_{i}=s, C_{i}=c, D_{i}=0\right)=(1-\mathrm{OD})\{F(y \mid s, c, 1)-F(y \mid s, c, 0) P(a \mid s, 0)\} /\{1-P(a \mid s, 0)\}+\mathrm{OD}$. 


\begin{tabular}{rrrrr}
\hline & $\mathrm{OD}=0.00$ & $\mathrm{OD}=0.33$ & $\mathrm{OD}=0.67$ & $\mathrm{OD}=1.00$ \\
\hline naïve & 0.002 & 0.011 & 0.020 & 0.030 \\
$\min$ & -0.001 & 0.001 & 0.001 & 0.001 \\
$\max$ & -0.001 & -0.002 & -0.002 & -0.001 \\
\hline
\end{tabular}

\begin{tabular}{rrrrr}
\hline $\mathrm{N}$ & $\mathrm{OD}=0.00$ & $\mathrm{OD}=0.33$ & $\mathrm{OD}=0.67$ & $\mathrm{OD}=1.00$ \\
\hline 500 & 0.959 & 0.967 & 0.970 & 0.962 \\
1000 & 0.956 & 0.966 & 0.971 & 0.968 \\
3000 & 0.946 & 0.954 & 0.950 & 0.968 \\
10000 & 0.938 & 0.954 & 0.958 & 0.966 \\
50000 & 0.946 & 0.954 & 0.954 & 0.946 \\
\hline
\end{tabular}

Table 3: Estimated Bias and Coverage Rates for Various OD Values, Holding CD at 0. The left table presents bias results for the naïve and the bounds lower/upper endpoint estimators, and the right table presents bounds posterior interval coverage rates. These results are based on the procedure of Table 2 , which presents the same quantities for varying $\mathrm{CD}$ values.

$\mathbf{1}\{F(y \mid s, a, 1)-F(y \mid s, a, 0) P(a \mid s, 0)<P(c \mid s, 0)\}\{F(y \mid s, a, 1)-F(y \mid s, a, 0) P(a \mid s, 0)\} / P(c \mid s, 0)$ for

a particular $c \neq a$, which then fixes the distribution of potential outcomes in the remaining choice-based subgroup. All values of the OD parameter produce observationally equivalent simulation populations, but the population ACTE differs depending on its setting. When $\mathrm{OD}=1$, the population ACTE is the most extreme effect consistent with the observable margins, lying on the endpoint of the population bounds. In contrast, $\mathrm{OD}=0$ produces the least extreme population satisfying the same observed margins, with $\operatorname{Pr}\left(Y_{i}(a) \leq y \mid S_{i}=s, C_{i}=c\right)$ identical to $\operatorname{Pr}\left(Y_{i}(a) \leq y \mid S_{i}=s, C_{i}=c^{\prime}\right)$. In these simulations, CD is set to zero.

Table 3 (left panel) presents the bias of naïve and bounds estimates at varying levels of the OD parameter, with sample sizes of 3,000. Once again, results demonstrate that while naïve estimates are accurate in a best-case simulation, bias is on the order of one standard error of any particular naïve estimate when assumptions are violated even moderately (OD between 0.33 and 0.67 ). Bias of the proposed bounds procedure remains negligible for all OD values. Table 3 (right panel) shows that coverage levels are as expected over a wide range for OD and $\mathrm{N}$, or perhaps slightly conservative at smaller sample sizes.

\section{Concluding Remarks}

Scholars of social and medical sciences have long sought to enhance the external validity of randomized experiments through various means. Medical researchers have often adopted PPTs to incorporate the preferences of experimental subjects over treatment options into their study designs, thereby tackling the question of what impact treatments have on the kinds of people who would actually take them if 
they were allowed to choose. However, systematic analysis of causal and statistical properties of PPTs has only just begun. In particular, researchers have largely neglected the potential discrepancy between subjects' stated and revealed preferences in the existing literature.

In this paper, we seek to address the challenge of improving external validity via a new experimental design for PPTs. The proposed design involves measurement of both stated preferences and actual choices as well as randomization into the standard RCT or a free-choice condition. The methodology we develop systematically addresses the potential inferential threat caused by nonignorable differences between stated and revealed preferences, using both nonparametric identification analysis and sensitivity analysis. As we illustrate in an original empirical example where we use the proposed framework, our method enables inference on a causal quantity of interest that captures the heterogeneity in treatment effects across revealed preferences without relying on the assumption of ignorable measurement error. We provide open-source software, ppt, which implements the proposed methodology.

Future statistical work on PPTs should investigate the consequence of noncompliance and differential attrition on the estimation of ACTEs, among other inferential challenges left unaddressed by the current paper. An important motivation for PPTs in medical research is the concern that a patient who strongly prefers one treatment option may not follow experimental protocols and cross over to another treatment arm or out of the study, damaging the internal validity of the experiment. Forcing patients into treatment options against their preferences may also be considered unethical in some applications (Lambert and Wood, 2000). One natural direction for future research is, therefore, to incorporate such complications under the current framework.

\section{References}

Arceneaux, K., Johnson, M., and Murphy, C. "Polarized Political Communication, Oppositional Media Hostility, and Selective Exposure.” Journal of Politics, 74(1):174-186 (2012).

Balke, A. "Probabilistic Counterfactuals: Semantics, Computation, and Applications." Ph.D. thesis, Computer Science Department, University of California, Los Angeles (1995). 
Balke, A. and Pearl, J. "Bounds on treatment effects from studies with imperfect compliance." Journal of the American Statistical Association, 92:1171-1176 (1997).

Brewin, C. R. and Bradley, C. "Patient preferences and randomised clinical trials." BMJ: British Medical Journal, 299(6694):313 (1989).

Brown, N. R. and Sinclair, R. C. "Estimating Number of Lifetime Sexual Partners: Men and Women Do It Differently." Journal of Sex Research, 36:292—297 (1999).

Campbell, A., Converse, P. E., Miller, W., and Stokes, D. The American Voter. Chicago: University of Chicago Press (1960).

Clausen, A. R. “Response Validity: Vote Report.” Public Opinion Quarterly, 32(4):588-606 (1968).

Frangakis, C. E. and Rubin, D. B. “Principal Stratification in Causal Inference.” Biometrics, 58(1):21-29 (2002).

Gaines, B. J. and Kuklinski, J. H. "Experimental Estimation of Heterogeneous Treatment Effects Related to Self-Selection.” American Journal of Political Science, 55(3):724-736 (2011).

Gallup. “Media Use and Evaluation.” Technical report, Gallup Historical Trends (2014). URL http: / / www.gallup.com/poll/1663/media-use-evaluation.aspx

Hamilton, J. T. "The Market and the Media." In Overholser, G. and Jamieson, K. H. (eds.), The Press. Oxford: Oxford University Press (2005).

Hirano, K., Imbens, G. W., Rubin, D. B., and Zhou, X.-H. "Assessing the effect of an influenza vaccine in an encouragement design.” Biostatistics, 1(1):69-88 (2000).

Horowitz, J. L. and Manski, C. F. "Nonparametric Analysis of Randomized Experiments With Missing Covariate and Outcome Data." Journal of the Americal Statistical Association, 95(449):77-84 (2000). 
Howard, L. and Thornicroft, G. "Patient preference randomised controlled trials in mental health research.” The British Journal of Psychiatry, 188(4):303-304 (2006).

Hser, Y.-i., Maglione, M., and Boyle, K. "Validity of Self-Report of Drug Use Among STD Patients, ER Patients, and Arrestees." American Journal of Drug and Alcohol Abuse, 25(1):81-91 (1999).

Imai, K., Tingley, D., and Yamamoto, T. "Experimental Designs for Identifying Causal Mechanisms (with discussions)." Journal of the Royal Statistical Society, Series A (Statistics in Society), 176(1):551 (2013).

Iyengar, S. and Hahn, K. S. "Red media, blue media: evidence of ideological selectivity in media use." Journal of Communication, 59:19-39 (2009).

Kim, Y. M. "Issue Publics in the New Information Environment: Selectivity, Domain Specificity, and Extremity." Communication Research, 36:254-284 (2009).

King, M., Nazareth, I., Lampe, F., Bower, P., Chandler, M., Morou, M., Sibbald, B., and Lai, R. "Impact of participant and physician intervention preferences on randomized trials: a systematic review." Jourmal of the American Medical Association, 293(9):1089-1099 (2005).

Kowalski, C. J. and Mrdjenovich, A. J. "Patient preference clinical trials: why and when they will sometimes be preferred." Perspectives in biology and medicine, 56(1):18-35 (2013).

Ladd, J. M. Why Americans Hate the Media and How It Matters. Princeton: Princeton University Press (2012).

Lambert, M. F. and Wood, J. "Incorporating patient preferences into randomized trials." Journal of clinical epidemiology, 53(2):163-166 (2000).

Levendusky, M. S. "Why Do Partisan Media Polarize Viewers?” American Journal of Political Science, 57(3):611-623 (2013). 
Long, Q., Little, R. J., and Lin, X. "Causal inference in hybrid intervention trials involving treatment choice." Journal of the American Statistical Association, 103(482):474-484 (2008).

Manski, C. F. Identification Problems in the Social Sciences. Harvard University Press (1995).

Neyman, J. "On the application of probability theory to agricultural experiments: Essay on principles, Section 9. (Translated in 1990)." Statistical Science, 5:465-480 (1923).

Payne, G. J. "The Bradley Effect: Mediated Reality of Race and Politics in the 2008 U.S. Presidential Election.” American Behavior Scientist, 54:417-435 (2010).

Prior, M. Post-Broadcast Democracy: How Media Choice Increases Inequality in Political Involvement and Polarizes Elections. Cambridge: Cambridge University Press (2007).

—. "The Immensely Inflated News Audience: Assessing Bias in Self-Reported News Exposure.” Public Opinion Quarterly, 73:1-14 (2009).

Rogers, T. and Aida, M. "Vote Self-Prediction Hardly Predicts Who Will Vote, And Is (Misleadingly) Unbiased." American Politics Research, 42(3):503-528 (2013).

Rosenbaum, P. R. Observational Studies. New York: Springer-Verlag, 2nd edition (2002).

Rubin, D. B. "Estimating Causal Effects of Treatments in Randomized and Nonrandomized Studies." Journal of Educational Psychology, 66(5):688-701 (1974).

—. "Comments on "On the Application of Probability Theory to Agricultural Experiments. Essay on Principles. Section 9" by J. Splawa-Neyman translated from the Polish and edited by D. M. Dabrowska and T. P. Speed.” Statistical Science, 5:472-480 (1990).

Schmoor, C., Olschewski, M., and Schumacher, M. "Randomized and non-randomized patients in clinical trials: experiences with comprehensive cohort studies." Statistics in medicine, 15(3):263-271 (1996). 
Stroud, N. J. The Politics of News Choice. Oxford: Oxford University Press (2011).

Torgerson, D. J., Klaber-Moffett, J., and Russell, I. T. "Patient preferences in randomised trials: threat or opportunity?" Journal of Health Services Research \& Policy, 1(4):194-197 (1996).

Tourangeau, R. "Remember What Happened: Memory Errors and Survey Reports." In Stone, A. A., Turkkan, J. S., Bachrach, C. A., Jobe, J. B., Kurtzman, H. S., and Cain, V. S. (eds.), Memory: The Science of Self Report: Implications for Research and Practice. Hove: Psychology Press (1999).

Tourangeau, R., Rips, L. J., and Rasinski, K. The Psychology of Survey Response. Cambridge University Press (2000).

Yamamoto, T. "Understanding the past: Statistical analysis of causal attribution." American Journal of Political Science, 56(1):237-256 (2012). 


\section{Supplementary Materials}

\section{A.1 Observable Implications of Assumption 3}

In this section, we derive the two observable implications of Assumption 3 described in Section 3.3 .

First, Assumption 3 implies,

$$
\mathbb{E}\left[Y_{i}(a) \mid C_{i}=a\right]=\mathbb{E}\left[Y_{i}(a) \mid S_{i}=a\right]
$$

for all $a \in \mathcal{A}$. This relationship directly implies equation (2) under Assumptions 1 and 2 . Second, note that equation 10 also implies,

$$
\begin{aligned}
& \mathbb{E}\left[Y_{i}(a) \mid C_{i}=a\right]=\mathbb{E}\left[Y_{i}(a) \mid C_{i}=a, S_{i}=a\right] \operatorname{Pr}\left(C_{i}=a \mid S_{i}=a\right) \\
&+ \mathbb{E}\left[Y_{i}(a) \mid C_{i} \neq a, S_{i}=a\right] \operatorname{Pr}\left(C_{i} \neq a \mid S_{i}=a\right) \\
& \Leftrightarrow \mathbb{E}\left[Y_{i}(a) \mid C_{i} \neq a, S_{i}=a\right]=\frac{\mathbb{E}\left[Y_{i} \mid C_{i}=a, D_{i}=0\right]-\mathbb{E}\left[Y_{i} \mid C_{i}=S_{i}=a, D_{i}=0\right] \operatorname{Pr}\left(C_{i}=a \mid S_{i}=a, D_{i}=0\right)}{1-\operatorname{Pr}\left(C_{i}=a \mid S_{i}=a, D_{i}=0\right)}
\end{aligned}
$$

for all $a \in \mathcal{A}$. Setting the unobserved term in the left-hand side to its theoretical maximum and minimum yields equation (3).

\section{A.2 Derivation of Equation (4)}

First, consider $\mathbb{E}\left[Y_{i}(a) \mid C_{i}=c\right]$. Assumptions 1 and 2 imply $\operatorname{Pr}\left(C_{i}=c, S_{i}=s\right)=\operatorname{Pr}\left(C_{i}=c, S_{i}=\right.$ $\left.s \mid D_{i}=0\right), \mathbb{E}\left[Y_{i}(c) \mid C_{i}=c, S_{i}=s\right]=\mathbb{E}\left[Y_{i} \mid C_{i}=c, S_{i}=s, D_{i}=0\right], \mathbb{E}\left[Y_{i}(a)\right]=\mathbb{E}\left[Y_{i} \mid A_{i}=a, D_{i}=1\right]$, and $\mathbb{E}\left[Y_{i}(a) \mid S_{i}=s\right]=\mathbb{E}\left[Y_{i} \mid S_{i}=s, A_{i}=a, D_{i}=1\right]$. Now, note that

$$
\mathbb{E}\left[Y_{i} \mid A_{i}=a, D_{i}=1\right]=\mathbb{E}\left[Y_{i}(a)\right]=\sum_{c^{\prime}=0}^{J-1} \mathbb{E}\left[Y_{i}(a) \mid C_{i}=c^{\prime}\right] \operatorname{Pr}\left(C_{i}=c^{\prime}\right),
$$

by Assumptions 1, 2 and the law of total expectation. Substituting observed outcomes from the freechoice group and rearranging terms, we have

$$
\mathbb{E}\left[Y_{i}(a) \mid C_{i}=c\right]=\frac{1}{\operatorname{Pr}\left(C_{i}=c \mid D_{i}=0\right)}\left\{\begin{array}{l}
\mathbb{E}\left[Y_{i} \mid A_{i}=a, D_{i}=1\right] \\
-\mathbb{E}\left[Y_{i} \mid C_{i}=a, D_{i}=0\right] \operatorname{Pr}\left(C_{i}=a \mid D_{i}=0\right) \\
-\sum_{c^{\prime} \notin\{a, c\}} \mathbb{E}\left[Y_{i}(a) \mid C_{i}=c^{\prime}\right] \operatorname{Pr}\left(C_{i}=c^{\prime} \mid D_{i}=0\right)
\end{array}\right\}
$$


because of Assumptions 1 and 2 . By the same token,

$$
\mathbb{E}\left[Y_{i}\left(a^{\prime}\right) \mid C_{i}=c\right]=\frac{1}{\operatorname{Pr}\left(C_{i}=c \mid D_{i}=0\right)}\left\{\begin{array}{l}
\mathbb{E}\left[Y_{i} \mid A_{i}=a^{\prime}, D_{i}=1\right] \\
-\mathbb{E}\left[Y_{i} \mid C_{i}=a^{\prime}, D_{i}=0\right] \operatorname{Pr}\left(C_{i}=a \mid D_{i}=0\right) \\
-\sum_{c^{\prime} \notin\left\{a^{\prime}, c\right\}} \mathbb{E}\left[Y_{i}\left(a^{\prime}\right) \mid C_{i}=c^{\prime}\right] \operatorname{Pr}\left(C_{i}=c^{\prime} \mid D_{i}=0\right)
\end{array}\right\}
$$

The quantity of interest is therefore

$$
\begin{aligned}
\tau\left(a, a^{\prime} \mid c\right)=\frac{1}{\operatorname{Pr}\left(C_{i}=c \mid D_{i}=0\right)}\left\{\begin{array}{l}
\mathbb{E}\left[Y_{i} \mid A_{i}=a, D_{i}=1\right] \\
-\mathbb{E}\left[Y_{i} \mid C_{i}=a, D_{i}=0\right] \operatorname{Pr}\left(C_{i}=a \mid D_{i}=0\right) \\
-\sum_{c^{\prime} \notin\{a, c\}} \mathbb{E}\left[Y_{i}(a) \mid C_{i}=c^{\prime}\right] \operatorname{Pr}\left(C_{i}=c^{\prime} \mid D_{i}=0\right)
\end{array}\right\} \\
-\frac{1}{\operatorname{Pr}\left(C_{i}=c \mid D_{i}=0\right)}\left\{\begin{array}{l}
\mathbb{E}\left[Y_{i} \mid A_{i}=a^{\prime}, D_{i}=1\right] \\
-\mathbb{E}\left[Y_{i} \mid C_{i}=a^{\prime}, D_{i}=0\right] \operatorname{Pr}\left(C_{i}=a^{\prime} \mid D_{i}=0\right) \\
-\sum_{c^{\prime} \notin\left\{a^{\prime}, c\right\}} \mathbb{E}\left[Y_{i}\left(a^{\prime}\right) \mid C_{i}=c^{\prime}\right] \operatorname{Pr}\left(C_{i}=c^{\prime} \mid D_{i}=0\right)
\end{array}\right\}
\end{aligned}
$$

for any $a, a^{\prime}$ and $c$. Thus, under Assumptions 1 and 2 , we have $2(J-2)$ terms that remain unidentified when $a \neq a^{\prime} \neq c$. When $a^{\prime}=c$, the above simplifies to

$$
\begin{aligned}
& \tau(a, c \mid c)= \mathbb{E}\left[Y_{i}(a) \mid C_{i}=c\right]-\mathbb{E}\left[Y_{i} \mid C_{i}=c, D_{i}=0\right] \\
&= \frac{1}{\operatorname{Pr}\left(C_{i}=c \mid D_{i}=0\right)}\left\{\begin{array}{l}
\mathbb{E}\left[Y_{i} \mid A_{i}=a, D_{i}=1\right] \\
-\mathbb{E}\left[Y_{i} \mid C_{i}=a, D_{i}=0\right] \operatorname{Pr}\left(C_{i}=a \mid D_{i}=0\right) \\
-\sum_{c^{\prime} \notin\{a, c\}} \mathbb{E}\left[Y_{i}(a) \mid C_{i}=c^{\prime}\right] \operatorname{Pr}\left(C_{i}=c^{\prime} \mid D_{i}=0\right)
\end{array}\right\} \\
&-\mathbb{E}\left[Y_{i} \mid C_{i}=c, D_{i}=0\right]
\end{aligned}
$$

and $J-2$ terms remain unidentified.

\section{A.3 Proof of Proposition 1}

We begin by establishing several lemmas.

Lemma .1 Let $\Gamma_{a}(y, c \mid s, a)=\operatorname{Pr}\left(Y_{i}(a) \leq y, C_{i} \leq c \mid S_{i}=s, C_{i} \neq a\right)$. Under Assumptions 1 and 2 , the sharp upper and lower bounds on $\Gamma_{a}(y, c \mid s, a)$, denoted by $\Gamma_{a}^{+}(y, c \mid s, a)$ and $\Gamma_{a}^{-}(y, c \mid s, a)$ respectively, are identified as follows.

$$
\begin{aligned}
\Gamma_{a}^{+}(y, c \mid s, a) & =\min \left\{H(c \mid s, a, 0), \frac{F(y \mid s, a, 1)-F(y \mid s, a, 0) P(a \mid s, 0)}{1-P(a \mid s, 0)}\right\} \\
\Gamma_{a}^{-}(y, c \mid s, a) & =\max \left\{0, H(c \mid s, a, 0)+\frac{F(y \mid s, a, 1)-F(y \mid s, a, 0) P(a \mid s, 0)}{1-P(a \mid s, 0)}-1\right\}
\end{aligned}
$$


for $y \in \mathcal{Y}, a, c, s \in \mathcal{A}$ and $d \in\{0,1\}$, where $H(c \mid s, a, 0)=\operatorname{Pr}\left(A_{i} \leq c \mid S_{i}=s, A_{i} \neq a, D_{i}=0\right)$ and $F(y \mid s, a, d)$ and $P(a \mid s, 0)$ are as defined in Proposition 1.

Proof. By the Fréchet-Hoeffding theorem, the sharp upper and lower bounds of the bivariate joint distribution function $\Gamma_{a}(y, c \mid s, a)$ are given by,

$$
\begin{aligned}
\Gamma_{a}^{+}(y, c \mid s, a) & =\min \left\{\Gamma_{a}(\infty, c \mid s, a), \Gamma_{a}(y, \infty \mid s, a)\right\}, \\
\Gamma_{a}^{-}(y, c \mid s, a) & =\max \left\{0, \Gamma_{a}(\infty, c \mid s, a)+\Gamma_{a}(y, \infty \mid s, a)-1\right\} .
\end{aligned}
$$

Under Assumption 1], $\Gamma_{a}(\infty, c \mid s, a)=\operatorname{Pr}\left(C_{i} \leq c \mid S_{i}=s, C_{i} \neq a\right)=\operatorname{Pr}\left(A_{i} \leq c \mid S_{i}=s, A_{i} \neq a, D_{i}=\right.$ $0)=H(c \mid s, a, 0)$ for any $c, s$ and $a \in \mathcal{A}$. Under Assumptions 1 and 2 , we have

$$
\begin{aligned}
\Gamma_{a}(y, \infty \mid s, a) & =\operatorname{Pr}\left(Y_{i}(a) \leq y \mid S_{i}=s, C_{i} \neq a\right) \\
& =\frac{\operatorname{Pr}\left(Y_{i}(a) \leq y \mid S_{i}=s\right)-\operatorname{Pr}\left(Y_{i}(a) \leq y, C_{i}=a \mid S_{i}=s\right)}{\operatorname{Pr}\left(C_{i} \neq a \mid S_{i}=s\right)} \\
& =\frac{\operatorname{Pr}\left(Y_{i}(a) \leq y \mid S_{i}=s\right)-\operatorname{Pr}\left(Y_{i}(a) \leq y \mid C_{i}=a, S_{i}=s\right) \operatorname{Pr}\left(C_{i}=a \mid S_{i}=s\right)}{1-\operatorname{Pr}\left(C_{i}=a \mid S_{i}=s\right)} \\
& =\frac{F(y \mid s, a, 1)-F(y \mid s, a, 0) P(a \mid s, 0)}{1-P(a \mid s, 0)}
\end{aligned}
$$

for any $a$ and $s \in \mathcal{A}$. Substituting these to equations (11) and (12) yields the results in Lemma 1.

Lemma .2 Let $A_{i}^{*}$, $C_{i}^{*}$ and $S_{i}^{*}$ be reordered versions of $A_{i}, C_{i}$ and $S_{i}$, respectively, such that $C_{i}^{*}=0$ iff $C_{i}=c\left(\right.$ and likewise for $A_{i}^{*}$ and $\left.S_{i}^{*}\right)$. Then, the resulting sharp bounds on $\Gamma_{a}(y, c \mid s, a)-\Gamma_{a}(y, c-1 \mid$ $s, a)$ are also the sharp bounds on $\Gamma_{a}^{*}(y, 0 \mid s, a)-\Gamma_{a}^{*}(y,-1 \mid s, a)$ for any y and $c \in \mathcal{A}$, where $\Gamma_{a}^{*}(y, c \mid s, a)=\operatorname{Pr}\left(Y_{i}(a) \leq y, C_{i}^{*} \leq c \mid S_{i}=s, C_{i} \neq a\right)$.

Proof. First, consider the sharp bounds on $\Gamma_{a}(y, c)-\Gamma_{a}(y, c-1)$. In addition to the Fréchet-Hoeffding constraints on its constituent parts,

$$
\begin{aligned}
\Gamma_{a}(y, c \mid s, a) & \in\left[\Gamma_{a}^{-}(y, c \mid s, a), \Gamma_{a}^{+}(y, c \mid s, a)\right] \\
\Gamma_{a}(y, c-1 \mid s, a) & \in\left[\Gamma_{a}^{-}(y, c-1 \mid s, a), \Gamma_{a}^{+}(y, c-1 \mid s, a)\right],
\end{aligned}
$$


the increase in cumulative probability from $c-1$ to $c$ is also subject to

$$
\Gamma_{a}(y, c)-\Gamma_{a}(y, c-1) \in\left[0, \Gamma_{a}(\infty, c)-\Gamma_{a}(\infty, c-1)\right] .
$$

The combination of these constraints yields

$$
\begin{aligned}
& \Gamma_{a}(y, c \mid s, a) \Gamma_{a}(y, c-1 \mid s, a) \\
& \in\left[0, \Gamma_{a}(\infty, c)-\Gamma_{a}(\infty, c-1)\right] \bigcup \\
& \left(\left[\Gamma_{a}^{*-}(y, c \mid s, a), \Gamma_{a}^{*+}(y, c \mid s, a)\right]-\left[\Gamma_{a}^{*-}(y, c-1 \mid s, a), \Gamma_{a}^{*+}(y, c-1 \mid s, a)\right]\right) \\
& \in\left[\operatorname { m a x } \left\{\max \left\{\begin{array}{l}
0, \\
\Gamma_{a}(\infty, c \mid s, a)+\Gamma_{a}(y, \infty \mid s, a)-1
\end{array}\right\}-\min \left\{\begin{array}{l}
\Gamma_{a}(\infty, c-1 \mid s, a), \\
\Gamma_{a}(y, \infty \mid s, a)
\end{array}\right\},\right.\right. \\
& \min \left\{\begin{array}{l}
\Gamma_{a}(\infty, c)-\Gamma_{a}(\infty, c-1), \\
\left.\left.\min \left\{\begin{array}{l}
\Gamma_{a}(\infty, c \mid s, a), \\
\Gamma_{a}(y, \infty \mid s, a)
\end{array}\right\}-\max \left\{\begin{array}{l}
0, \\
\Gamma_{a}(\infty, c-1 \mid s, a)+\Gamma_{a}(y, \infty \mid s, a)-1
\end{array}\right\}\right)\right]
\end{array}\right.
\end{aligned}
$$

Next, consider the sharp bounds on $\Gamma_{a}^{*}(y, 0 \mid s, a)-\Gamma_{a}^{*}(y,-1 \mid s, a)$. Because -1 lies below the lowest possible value of $C_{i}^{*}, \Gamma_{a}^{*}(y,-1 \mid s, a)$ is necessarily zero, and bounds on the difference reduce to bounds on $\Gamma_{a}^{*}(y, 0 \mid s, a)$,

$$
\begin{aligned}
\Gamma_{a}^{*}(y, 0 \mid s, a) \in & {\left[\Gamma_{a}^{*-}(y, 0 \mid s, a), \Gamma_{a}^{*+}(y, 0 \mid s, a)\right] } \\
\in & {\left[\max \left\{0, \Gamma_{a}^{*}(\infty, 0 \mid s, a)+\Gamma_{a}^{*}(y, \infty \mid s, a)-1\right\}, \min \left\{\Gamma_{a}^{*}(\infty, 0 \mid s, a), \Gamma_{a}^{*}(y, \infty \mid s, a)\right\}\right] } \\
\in & {\left[\max \left\{0, \operatorname{Pr}\left(A_{i}=c \mid S_{i}=s, A_{i} \neq a, D_{i}=0\right)+\Gamma_{a}(y, \infty \mid s, a)-1\right\},\right.} \\
& \left.\min \left\{\operatorname{Pr}\left(A_{i}=c \mid S_{i}=s, A_{i} \neq a, D_{i}=0\right), \Gamma_{a}(y, \infty \mid s, a)\right\}\right] \\
\in & {\left[\max \left\{0, \Gamma_{a}(\infty, c \mid s, a)-\Gamma_{a}(\infty, c-1 \mid s, a)+\Gamma_{a}(y, \infty \mid s, a)-1\right\},\right.} \\
& \left.\min \left\{\Gamma_{a}(\infty, c \mid s, a)-\Gamma_{a}(\infty, c-1 \mid s, a), \Gamma_{a}(y, \infty \mid s, a)\right\}\right] .
\end{aligned}
$$

We now show that the upper bound on $\Gamma_{a}(y, c)-\Gamma_{a}(y, c-1)$ is identical to the upper bound on 
$\Gamma_{a}^{*}(y, 0 \mid s, a)-\Gamma_{a}^{*}(y,-1 \mid s, a)$ in each of the following four possible cases. (1) $\Gamma_{a}(\infty, c \mid s, a) \leq$ $\Gamma_{a}(y, \infty \mid s, a)$ and $0 \geq \Gamma_{a}(\infty, c-1 \mid s, a)+\Gamma_{a}(y, \infty \mid s, a)-1$. The upper bound on $\Gamma_{a}(y, c)-\Gamma_{a}(y, c-1)$ reduces to $\min \left\{\Gamma_{a}(\infty, c)-\Gamma_{a}(\infty, c-1), \Gamma_{a}(\infty, c \mid s, a)-\max \left\{0, \Gamma_{a}(\infty, c-1 \mid s, a)+\Gamma_{a}(y, \infty \mid s, a)-1\right\}\right\}$. This implies $\Gamma_{a}(\infty, c \mid s, a)-\Gamma_{a}(\infty, c-1 \mid s, a) \leq \Gamma_{a}(y, \infty \mid s, a)$, and so the upper bound on $\Gamma_{a}^{*}(y, 0 \mid s, a)$ becomes $\Gamma_{a}(\infty, c \mid s, a)-\Gamma_{a}(\infty, c-1 \mid s, a)$. Since $0 \geq \Gamma_{a}(\infty, c-1 \mid s, a)+\Gamma_{a}(y, \infty \mid s, a)-1$, the upper bound on $\Gamma_{a}(y, c)-\Gamma_{a}(y, c-1)$ further reduces to $\min \left\{\Gamma_{a}(\infty, c)-\Gamma_{a}(\infty, c-1), \Gamma_{a}(\infty, c \mid s, a)\right\}=$ $\Gamma_{a}(\infty, c)-\Gamma_{a}(\infty, c-1)$, which is identical to the upper bound on $\Gamma_{a}^{*}(y, 0 \mid s, a)$. (2) $\Gamma_{a}(\infty, c \mid s, a) \leq$ $\Gamma_{a}(y, \infty \mid s, a)$ and $0<\Gamma_{a}(\infty, c-1 \mid s, a)+\Gamma_{a}(y, \infty \mid s, a)-1$. The upper bound on $\Gamma_{a}(y, c)-\Gamma_{a}(y, c-1)$ becomes $\min \left\{\Gamma_{a}(\infty, c)-\Gamma_{a}(\infty, c-1), \Gamma_{a}(\infty, c \mid s, a)-\left(\Gamma_{a}(\infty, c-1 \mid s, a)+\Gamma_{a}(y, \infty \mid s, a)-1\right)\right\}=$ $\Gamma_{a}(\infty, c)-\Gamma_{a}(\infty, c-1)$, since $1-\Gamma_{a}(y, \infty \mid s, a)>0$. This is again identical to the upper bound on $\Gamma_{a}^{*}(y, 0 \mid s, a)$. (3) $\Gamma_{a}(\infty, c \mid s, a)>\Gamma_{a}(y, \infty \mid s, a)$ and $0 \geq \Gamma_{a}(\infty, c-1 \mid s, a)+\Gamma_{a}(y, \infty \mid s, a)-1$. The upper bound on $\Gamma_{a}(y, c)-\Gamma_{a}(y, c-1)$ reduces to $\min \left\{\Gamma_{a}(\infty, c)-\Gamma_{a}(\infty, c-1), \Gamma_{a}(y, \infty \mid s, a)-\right.$ $\left.\max \left\{0, \Gamma_{a}(\infty, c-1 \mid s, a)+\Gamma_{a}(y, \infty \mid s, a)-1\right\}\right\}$. Since $0 \geq \Gamma_{a}(\infty, c-1 \mid s, a)+\Gamma_{a}(y, \infty \mid s, a)-1$, the upper bound on $\Gamma_{a}(y, c)-\Gamma_{a}(y, c-1)$ further reduces to $\min \left\{\Gamma_{a}(\infty, c)-\Gamma_{a}(\infty, c-1), \Gamma_{a}(y, \infty \mid s, a)\right\}$, which is the original upper bound given for $\Gamma_{a}^{*}(y, 0 \mid s, a)$. (4) $\Gamma_{a}(\infty, c \mid s, a)>\Gamma_{a}(y, \infty \mid s, a)$ and $0<\Gamma_{a}(\infty, c-1 \mid s, a)+\Gamma_{a}(y, \infty \mid s, a)-1$. The upper bound on $\Gamma_{a}(y, c)-\Gamma_{a}(y, c-1)$ further reduces to $\min \left\{\Gamma_{a}(\infty, c)-\Gamma_{a}(\infty, c-1), 1-\Gamma_{a}(\infty, c-1 \mid s, a)\right\}=\Gamma_{a}(\infty, c)-\Gamma_{a}(\infty, c-1)$. This implies that $\Gamma_{a}(\infty, c \mid s, a)-\Gamma_{a}(\infty, c-1 \mid s, a)<\Gamma_{a}(y, \infty \mid s, a)$. The upper bound on $\Gamma_{a}^{*}(y, 0 \mid s, a)$ then also becomes $\Gamma_{a}(\infty, c \mid s, a)-\Gamma_{a}(\infty, c-1 \mid s, a)$.

Finally, we show that the lower bound on $\Gamma_{a}(y, c)-\Gamma_{a}(y, c-1)$ is identical to the upper bound on $\Gamma_{a}^{*}(y, 0 \mid s, a)-\Gamma_{a}^{*}(y,-1 \mid s, a)$ in each of the following three possible cases. (1) $0 \geq \Gamma_{a}(\infty, c \mid s, a)+$ $\Gamma_{a}(y, \infty \mid s, a)-1$. The lower bound on $\Gamma_{a}(y, c)-\Gamma_{a}(y, c-1)$ reduces to $\max \left\{0,-\min \left\{\Gamma_{a}(\infty, c-\right.\right.$ $\left.\left.1 \mid s, a), \Gamma_{a}(y, \infty \mid s, a)\right\}\right\}=0$. Because $\Gamma_{a}(\infty, c \mid s, a) \geq \Gamma_{a}(\infty, c \mid s, a)-\Gamma_{a}(\infty, c-1 \mid s, a)$, the lower bound on $\Gamma_{a}^{*}(y, 0 \mid s, a)$ also becomes 0 . (2) $0<\Gamma_{a}(\infty, c \mid s, a)+\Gamma_{a}(y, \infty \mid s, a)-1$ and $\Gamma_{a}(\infty, c-$ $1 \mid s, a) \leq \Gamma_{a}(y, \infty \mid s, a)$. The lower bound on $\Gamma_{a}(y, c)-\Gamma_{a}(y, c-1)$ reduces to $\max \left\{0, \Gamma_{a}(\infty, c \mid s, a)+\right.$ $\left.\Gamma_{a}(y, \infty \mid s, a)-1-\min \left\{\Gamma_{a}(\infty, c-1 \mid s, a), \Gamma_{a}(y, \infty \mid s, a)\right\}\right\}$. Since $\Gamma_{a}(\infty, c-1 \mid s, a) \leq \Gamma_{a}(y, \infty \mid s, a)$, 
the lower bound on $\Gamma_{a}(y, c)-\Gamma_{a}(y, c-1)$ reduces further to $\max \left\{0, \Gamma_{a}(\infty, c \mid s, a)+\Gamma_{a}(y, \infty \mid s, a)-1-\right.$ $\left.\Gamma_{a}(\infty, c-1 \mid s, a)\right\}$, which is the original lower bound given for $\Gamma_{a}^{*}(y, 0 \mid s, a)$. (3) $0<\Gamma_{a}(\infty, c \mid s, a)+$ $\Gamma_{a}(y, \infty \mid s, a)-1$ and $\Gamma_{a}(\infty, c-1 \mid s, a)>\Gamma_{a}(y, \infty \mid s, a)$. The lower bound on $\Gamma_{a}(y, c)-\Gamma_{a}(y, c-1)$ reduces further to $\max \left\{0, \Gamma_{a}(\infty, c \mid s, a)+\Gamma_{a}(y, \infty \mid s, a)-1-\Gamma_{a}(y, \infty \mid s, a)\right\}=0$. Since $\Gamma_{a}(\infty, c \mid s, a)-$ $\Gamma_{a}(\infty, c-1 \mid s, a)+\Gamma_{a}(y, \infty \mid s, a)-1<\Gamma_{a}(\infty, c \mid s, a)-\Gamma_{a}(y, \infty \mid s, a)+\Gamma_{a}(y, \infty \mid s, a)-1<0$ and $\Gamma_{a}(\infty, c \mid s, a)-1 \leq 0$, the lower bound for $\Gamma_{a}^{*}(y, 0 \mid s, a)$ is also zero.

Lemma .3 Let $\Phi_{a}(y \mid s, c)=\operatorname{Pr}\left(Y_{i}(a) \leq y \mid S_{i}=s, C_{i}=c\right)$. Under Assumptions 1 and 2 the sharp upper and lower bounds on $\Phi_{a}(y \mid s, 0)$, denoted by $\Phi_{a}^{+}(y \mid s, 0)$ and $\Phi_{a}^{-}(y \mid s, 0)$ respectively, are identified as

$$
\begin{aligned}
& \Phi_{a}^{+}(y \mid s, 0)=\min \left\{1, \frac{F(y \mid s, a, 1)-F(y \mid s, a, 0) P(a \mid s, 0)}{P(0 \mid s, 0)}\right\} \text { and } \\
& \Phi_{a}^{-}(y \mid s, 0)=\max \left\{0,1+\frac{P(a \mid s, 0)+F(y \mid s, a, 1)-F(y \mid s, a, 0) P(a \mid s, 0)-1}{P(0 \mid s, 0)}\right\}
\end{aligned}
$$

for $y \in \mathcal{Y}$ and $a, s \in \mathcal{A}$.

Proof. First, note that

$$
\begin{aligned}
\Phi_{a}(y \mid s, c) & =\operatorname{Pr}\left(Y_{i}(a) \leq y \mid S_{i}=s, C_{i}=c, C_{i} \neq a\right) \\
& =\frac{\operatorname{Pr}\left(Y_{i}(a) \leq y, C_{i} \leq c \mid S_{i}=s, C_{i} \neq a\right)-\operatorname{Pr}\left(Y_{i}(a) \leq y, C_{i} \leq c-1 \mid S_{i}=s, C_{i}=\neq a\right)}{\operatorname{Pr}\left(C_{i}=c \mid S_{i}=s, C_{i} \neq a\right)} \\
& =\frac{\Gamma_{a}(y, c \mid s, a)-\Gamma_{a}(y, c-1 \mid s, a)}{\operatorname{Pr}\left(C_{i}=c \mid S_{i}=s, C_{i} \neq a\right)},
\end{aligned}
$$

for $c \neq a$. By Lemma .1 , the sharp upper and lower bounds on $\Phi_{a}(y \mid s, c)$ are given by

$$
\begin{aligned}
\Phi_{a}^{+}(y \mid s, c) & =\min \left\{1, \frac{\Gamma_{a}^{+}(y, c \mid s, a)-\Gamma_{a}^{-}(y, c-1 \mid s, a)}{\operatorname{Pr}\left(C_{i}=c \mid S_{i}=s, C_{i} \neq a\right)}\right\}, \\
\Phi_{a}^{-}(y \mid s, c) & =\max \left\{0, \frac{\Gamma_{a}^{-}(y, c \mid s, a)-\Gamma_{a}^{+}(y, c-1 \mid s, a)}{\operatorname{Pr}\left(C_{i}=c \mid S_{i}=s, C_{i} \neq a\right)}\right\} .
\end{aligned}
$$

Because $\Gamma_{a}^{+}(y,-1 \mid s, a)=\Gamma_{a}^{-}(y,-1 \mid s, a)=0$ and by Lemma 1 , these bounds simplify when $c=0$ to

$$
\Phi_{a}^{+}(y \mid s, 0)=\frac{\Gamma_{a}^{+}(y, 0 \mid s, a)}{\operatorname{Pr}\left(C_{i}=0 \mid S_{i}=s, C_{i} \neq a\right)}
$$




$$
\begin{aligned}
& =\min \left\{\frac{H(0 \mid s, a, 0)}{\operatorname{Pr}\left(C_{i}=0 \mid S_{i}=s, C_{i} \neq a\right)}, \frac{F(y \mid s, a, 1)-F(y \mid s, a, 0) P(a \mid s, 0)}{\operatorname{Pr}\left(C_{i}=0 \mid S_{i}=s, C_{i} \neq a\right)\{1-P(a \mid s, 0)\}}\right\} \\
& =\min \left\{\frac{H(0 \mid s, a, 0)}{\operatorname{Pr}\left(A_{i}=0 \mid S_{i}=s, A_{i} \neq a, D_{i}=0\right)},\right. \\
& \left.\frac{F(y \mid s, a, 1)-F(y \mid s, a, 0) P(a \mid s, 0)}{\operatorname{Pr}\left(A_{i}=0 \mid S_{i}=s, A_{i} \neq a, D_{i}=0\right) \operatorname{Pr}\left(A_{i} \neq a \mid S_{i}=s, D_{i}=0\right)}\right\} \\
& =\min \left\{1, \frac{F(y \mid s, a, 1)-F(y \mid s, a, 0) P(a \mid s, 0)}{\operatorname{Pr}\left(A_{i}=0 \mid S_{i}=s, D_{i}=0\right)}\right\}
\end{aligned}
$$

and

$$
\begin{aligned}
\Phi_{a}^{-}(y \mid s, 0) & =\frac{\Gamma_{a}^{-}(y, 0 \mid s, a)}{\operatorname{Pr}\left(C_{i}=0 \mid S_{i}=s, C_{i} \neq a\right)} \\
& =\max \left\{0, \frac{F(y \mid s, a, 1)-F(y \mid s, a, 0) P(a \mid s, 0)-\{1-H(0 \mid s, a, 0)\}\{1-P(a \mid s, 0)\}}{\operatorname{Pr}\left(C_{i}=0 \mid S_{i}=s, C_{i} \neq a\right)\{1-P(a \mid s, 0)\}}\right\} \\
& =\max \left\{0, \frac{F(y \mid s, a, 1)-F(y \mid s, a, 0) P(a \mid s, 0)-1+P(a \mid s, 0)}{\operatorname{Pr}\left(A_{i}=0 \mid S_{i}=s, D_{i}=0\right)}+1\right\} .
\end{aligned}
$$

Now we provide a proof for the bounds in Proposition 1. We only consider the case of $c=0$. This can be done without loss of generality by Lemma .2 . Now, note that $\tau\left(a, a^{\prime} \mid 0\right)$ can be written under Assumption 1 as,

$$
\tau\left(a, a^{\prime} \mid 0\right)=\sum_{s \in \mathcal{A}}\left\{\pi(a \mid s, 0)-\pi\left(a^{\prime} \mid s, 0\right)\right\} \operatorname{Pr}\left(S_{i}=s \mid A_{i}=0, D_{i}=0\right)
$$

where $\pi(a \mid s, c) \equiv \mathbb{E}\left[Y_{i}(a) \mid S_{i}=s, C_{i}=c\right]$ for any $a$ and $c \in \mathcal{A}$. Under Assumption $1, \pi(a \mid s, 0)$ can be point-identified when $a=0$ as

$$
\pi(0 \mid s, 0)=\mathbb{E}\left[Y_{i} \mid A_{i}=0, S_{i}=s, D_{i}=0\right],
$$

for any $s \in \mathcal{A}$, but not when $a \neq 0$. To find the sharp bounds on $\pi(a \mid s, 0)$ when $a \neq 0$, note that

$$
\pi(a \mid s, 0)=\lim _{y^{*} \rightarrow-\infty}\left\{\int_{y^{*}}^{\infty} 1-\Phi_{a}(y \mid s, 0) \mathrm{d} y+y^{*}\right\} .
$$

By Lemma $.3, \pi^{-}(a \mid s, 0) \leq \pi(a \mid s, 0) \leq \pi^{+}(a \mid s, 0)$ where

$$
\begin{aligned}
& \pi^{-}(a \mid s, 0) \equiv \lim _{y^{*} \rightarrow-\infty}\left\{\int_{y^{*}}^{\infty} 1-\Phi_{a}^{+}(y \mid s, 0) \mathrm{d} y+y^{*}\right\}, \\
& \pi^{+}(a \mid s, 0) \equiv \lim _{y^{*} \rightarrow-\infty}\left\{\int_{y^{*}}^{\infty} 1-\Phi_{a}^{-}(y \mid s, 0) \mathrm{d} y+y^{*}\right\} .
\end{aligned}
$$


The bounds, $\pi^{-}(a \mid s, 0)$ and $\pi^{+}(a \mid s, 0)$, are the sharp lower and upper bounds on $\pi(a \mid s, 0)$ because $\Phi_{a}^{+}(y \mid s, 0)$ and $\Phi_{a}^{-}(y \mid s, 0)$ are the sharp upper and lower bounds on $\Phi_{a}(y \mid s, 0)$, respectively.

Substituting Equations (14), (15) and (16) into Equation (13) and simplifying the terms yield the sharp bounds on $\tau(a, 0 \mid 0)$,

$$
\begin{gathered}
\sum_{s \in \mathcal{A}}\left\{\pi^{-}(a \mid s, 0) \operatorname{Pr}\left(S_{i}=s \mid A_{i}=0, D_{i}=0\right)\right\}-\mathbb{E}\left[Y_{i} \mid A_{i}=0, D_{i}=0\right] \\
\leq \tau(a, 0 \mid 0) \leq \\
\sum_{s \in \mathcal{A}}\left\{\pi^{+}(a \mid s, 0) \operatorname{Pr}\left(S_{i}=s \mid A_{i}=0, D_{i}=0\right)\right\}-\mathbb{E}\left[Y_{i} \mid A_{i}=0, D_{i}=0\right]
\end{gathered}
$$

for any $a \in \mathcal{A}$. For $\tau\left(a, a^{\prime}\right)$ where $a \neq a^{\prime}$, we obtain the following bounds,

$$
\begin{gathered}
\sum_{s \in \mathcal{A}}\left\{\pi^{-}(a \mid s, 0)-\pi^{+}\left(a^{\prime} \mid s, 0\right)\right\} \operatorname{Pr}\left(S_{i}=s \mid A_{i}=0, D_{i}=0\right) \\
\leq \tau\left(a, a^{\prime} \mid 0\right) \leq \\
\sum_{s \in \mathcal{A}}\left\{\pi^{+}(a \mid s, 0)-\pi^{-}\left(a^{\prime} \mid s, 0\right)\right\} \operatorname{Pr}\left(S_{i}=s \mid A_{i}=0, D_{i}=0\right)
\end{gathered}
$$

which are not necessarily sharp because $\pi^{-}(a \mid s, 0)$ and $\pi^{+}\left(a^{\prime} \mid s, 0\right)$ may not be simultaneously attainable, and vice versa. Finally, Lemma .2 implies that (17) and (18) are both valid as bounds for $\tau(a, c \mid c)$ and $\tau\left(a, a^{\prime} \mid c\right)$, respectively, for any $\mathrm{c} \in \mathcal{A}$. This completes the proof of Proposition 1

\section{A.4 Proof of Proposition 2}

We begin by considering the joint distribution of all variables in the study population when $J=3$ :

$$
\begin{aligned}
\operatorname{Pr}\left(S_{i}=\right. & \left.s, D_{i}=d, C_{i}=c, A_{i}=a, Y_{i}=y, Y_{i}(0)=y_{0}, Y_{i}(1)=y_{1}, Y_{i}(2)=y_{2}\right) \\
=\operatorname{Pr} & \left.Y_{i}(d)=y \mid A_{i}=a, Y_{i}(0)=y_{0}, Y_{i}(1)=y_{1}, Y_{i}(2)=y_{2}\right) \\
\quad & \operatorname{Pr}\left(A_{i}=a \mid C_{i}=c, D_{i}=d\right) \\
\quad & \operatorname{Pr}\left(S_{i}=s, C_{i}=c, Y_{i}(0)=y_{0}, Y_{i}(1)=y_{1}, Y_{i}(2)=y_{2}\right) \operatorname{Pr}\left(D_{i}=d\right) \\
= & \operatorname{Pr}\left(Y_{i}(d)=y \mid A_{i}=a, Y_{i}(0)=y_{0}, Y_{i}(1)=y_{1}, Y_{i}(2)=y_{2}\right) \\
\quad & \times\left\{\operatorname{Pr}\left(A_{i}=a \mid C_{i}=c, D_{i}=0\right)(1-d)+\operatorname{Pr}\left(A_{i}=a \mid D_{i}=1\right) d\right\}
\end{aligned}
$$




$$
\times \operatorname{Pr}\left(S_{i}=s, C_{i}=c, Y_{i}(0)=y_{0}, Y_{i}(1)=y_{1}, Y_{i}(2)=y_{2}\right) \operatorname{Pr}\left(D_{i}=d\right)
$$

where the first equality follows from Assumption 1 and the fact that $Y_{i}(0), Y_{i}(1), Y_{i}(2)$ and $A_{i}$ are sufficient for $Y_{i}$ and that $C_{i}$ and $D_{i}$ are sufficient for $A_{i}$. The second equality is by Assumption 2, Note that $\operatorname{Pr}\left(Y_{i}(d)=y \mid Y_{i}(0), Y_{i}(1), Y_{i}(2)\right)$ and $\operatorname{Pr}\left(A_{i}=a \mid C_{i}, D_{i}=0\right)$ are degenerate and that $\operatorname{Pr}\left(A_{i}=\right.$ $\left.a \mid D_{i}=1\right)$ and $\operatorname{Pr}\left(D_{i}=d\right)$ are fixed by the experimental design. Therefore, the remaining component of equation (19), $\operatorname{Pr}\left(S_{i}=s, C_{i}=c, Y_{i}(0)=y_{0}, Y_{i}(1)=y_{1}, Y_{i}(2)=y_{2}\right)$, completely specifies the data generating process, with $|\mathcal{A}|^{2} \cdot|\mathcal{Y}|^{|\mathcal{A}|}-1=J^{2} 2^{J}-1$ free parameters needed to describe it. Balke 1995 , Section 3.5) shows that bounds on counterfactual probabilities found by optimizing over such a complete model are sharp; that is, they are guaranteed to be at least as tight as bounds calculated from any partial (marginalized) model.

We express the complete model in terms of $\phi_{y_{0}, y_{1}, y_{2}, s, c} \in \Phi$. First, note that $\sum_{y_{0} \in\{0,1\}} \sum_{y_{1} \in\{0,1\}}$ $\sum_{y_{2} \in\{0,1\}} \sum_{s^{\prime} \in \mathcal{A}} \sum_{c^{\prime} \in \mathcal{A}} \phi_{y_{a}, y_{a^{\prime}}, y_{a^{\prime \prime}}, s^{\prime}, c^{\prime}}=1$. Next, from the free-choice condition, we observe $\operatorname{Pr}\left(S_{i}=\right.$ $s, C_{i}=c, Y_{i}=y \mid D_{i}=0$ ), which is completely specified by $|\mathcal{A}|^{2} \cdot|\mathcal{Y}|-1=2 J^{2}-1$ free parameters. We use the following $2 J^{2}$ marginals as constraints on $\phi_{y_{0}, y_{1}, y_{2}, s, c}$ (with one redundant):

$$
\begin{array}{r}
\operatorname{Pr}\left(S_{i}=s, A_{i}=c \mid D_{i}=0\right)=\operatorname{Pr}\left(S_{i}=s, C_{i}=c\right)=\sum_{a \in \mathcal{A}} \sum_{y_{a} \in\{0,1\}} \phi_{y_{0}, y_{1}, y_{2}, s, c} \\
\operatorname{Pr}\left(S_{i}=s, A_{i}=c, Y_{i}=1 \mid D_{i}=0\right)=\operatorname{Pr}\left(S_{i}=s, C_{i}=c, Y_{i}(c)=1\right)=\sum_{a \neq c} \sum_{y_{a} \in\{0,1\}} \phi_{y_{0}, y_{1}, y_{2}, s, c}
\end{array}
$$

for all $s$ and $c \in \mathcal{A}$. Similarly, from the forced-choice condition, we observe

$$
\begin{aligned}
\operatorname{Pr}\left(S_{i}\right. & \left.=s, A_{i}=a, Y_{i}=y \mid D_{i}=1\right) \\
& =\operatorname{Pr}\left(Y_{i}=y \mid S_{i}=s, A_{i}=a, D_{i}=1\right) \operatorname{Pr}\left(A_{i}=a \mid D_{i}=1\right) \operatorname{Pr}\left(S_{i}=s \mid D_{i}=1\right)
\end{aligned}
$$

where the equality holds by Assumption 2 Because $\operatorname{Pr}\left(A_{i}=a \mid D_{i}=1\right)$ is fixed a priori by randomization, the observed distribution from the forced-choice arm can be fully characterized by $(|\mathcal{Y}|-1)|\mathcal{A}|^{2}+$ $|\mathcal{A}|-1=J^{2}+J-1$ free parameters. We use the following $J^{2}+J$ margins as constraints on $\phi_{y_{0}, y_{1}, y_{2}, s, c}$, 
noting that one of them is redundant:

$$
\begin{aligned}
& \operatorname{Pr}\left(S_{i}=s \mid A_{i}=a, D_{i}=1\right)=\operatorname{Pr}\left(S_{i}=s\right)=\sum_{a \in \mathcal{A}} \sum_{y_{a} \in\{0,1\}} \sum_{c \in \mathcal{A}} \phi_{y_{0}, y_{1}, y_{2}, s, c}, \\
& \operatorname{Pr}\left(S_{i}=s, Y_{i}=1 \mid A_{i}=a, D_{i}=1\right)=\operatorname{Pr}\left(S_{i}=s, Y_{i}(a)=1\right)=\sum_{a^{\prime} \in \mathcal{A}} \sum_{y_{a^{\prime}} \in\{0,1\}} \sum_{c \in \mathcal{A}} \phi_{y_{0}, y_{1}, y_{2}, s, c} \cdot \mathbf{1}\left\{y_{a}=1\right\},
\end{aligned}
$$

for all $s$ and $a \in \mathcal{A}$. However, note that equation (22) are merely linear combinations of equation (20) and can therefore be omitted.

Finally, the quantity of interest can be written in terms of $\phi_{y_{0}, y_{1}, y_{2}, s, c}$ as,

$$
\begin{aligned}
\tau\left(a, a^{\prime} \mid c\right) & =\mathbb{E}\left[Y_{i}(a) \mid C_{i}=c\right]-\mathbb{E}\left[Y_{i}\left(a^{\prime}\right) \mid C_{i}=c\right] \\
& =\frac{\sum_{y_{0} \in\{0,1\}} \sum_{y_{2} \in\{0,1\}} \sum_{s} \phi_{1, y_{1}, y_{2}, s, c}}{\operatorname{Pr}\left(A_{i}=c \mid D_{i}=0\right)}-\frac{\sum_{y_{1} \in\{0,1\}} \sum_{y_{2} \in\{0,1\}} \sum_{s} \phi_{y_{0}, 1, y_{2}, s, c}}{\operatorname{Pr}\left(A_{i}=c \mid D_{i}=0\right)}
\end{aligned}
$$

assuming $a^{\prime}=1$ and $a=0$ without loss of generality. Solving for the extrema of $\tau\left(a, a^{\prime} \mid c\right)$ under the above set of linear constraints, which incorporate the full information in the observed data as well as probability axioms, yields its sharp upper and lower bounds as displayed in Proposition 2 .

\section{A.5 Statistical Inference for the Bounds}

Let $\boldsymbol{p}=\left[p_{s}\right]=\left[\operatorname{Pr}\left(S_{i}=0\right), \cdots, \operatorname{Pr}\left(S_{i}=J-1\right)\right]^{\top}$ be a stochastic vector of stated-preference probabilities. $\boldsymbol{q}=\left[q_{s c}\right]=\left[\operatorname{Pr}\left(C_{i}=c \mid S_{i}=s\right)\right]$ is a row-stochastic matrix, where row $s$, denoted $\boldsymbol{q}_{s}$, represents the distribution of true preferences $\left(C_{i}\right)$ among those with the stated preference $S_{i}=$ s. Also let $\boldsymbol{\pi}^{+}=\left\{\pi^{+}(a \mid s, c): a, s, c \in \mathcal{A}\right\}$ and $\boldsymbol{\pi}^{-}=\left\{\pi^{-}(a \mid s, c): a, s, c \in \mathcal{A}\right\}$, where $\pi^{+}(a \mid s, c)$ and $\pi^{-}(a \mid s, c)$ are defined in Appendix A.3. Let $\boldsymbol{F}^{1}=\{F(y \mid s, a, d): s, a \in \mathcal{A}, d=1\}$ and $\boldsymbol{F}^{0}=$ $\{F(y \mid s, a, d): s, a \in \mathcal{A}, d=0\}$, where $F(y \mid s, a, d)$ is defined in Proposition 1 . Finally, we use $\boldsymbol{\tau}^{+}$and $\boldsymbol{\tau}^{-}$to denote the sets of the upper and lower bounds on $\tau\left(a, a^{\prime} \mid c\right)$ for all $a, a^{\prime}, c \in \mathcal{A}$, respectively, and $\boldsymbol{X}$ to indicate all observed data.

Our goal is to approximate the posterior distribution of $\left(\boldsymbol{\tau}^{-}, \boldsymbol{\tau}^{+}\right)$with Monte Carlo simulations. We begin by the general bounds in Proposition 1 . Note that $\tau^{-}$and $\tau^{+}$are deterministic functions of $\boldsymbol{\pi}^{-}$, 
$\boldsymbol{\pi}^{+}, \boldsymbol{p}$ and $\boldsymbol{q}$, such that

$$
\begin{aligned}
\tau^{-}\left(a, a^{\prime} \mid c\right) & =\sum_{s \in \mathcal{A}}\left(\pi^{-}(a \mid s, c)-\pi^{+}\left(a^{\prime} \mid s, c\right)\right) \frac{q_{s c} p_{s}}{\sum_{s^{\prime} \in \mathcal{A}} q_{s^{\prime} c} p_{s^{\prime}}} \\
\tau^{+}\left(a, a^{\prime} \mid c\right) & =\sum_{s \in \mathcal{A}}\left(\pi^{+}(a \mid s, c)-\pi^{-}\left(a^{\prime} \mid s, c\right)\right) \frac{q_{s c} p_{s}}{\sum_{s^{\prime} \in \mathcal{A}} q_{s^{\prime} c} p_{s^{\prime}}}
\end{aligned}
$$

for all $a, a^{\prime}, c \in \mathcal{A}$. Therefore, we consider the problem of simulating samples from the joint posterior of $\boldsymbol{\pi}^{-}, \boldsymbol{\pi}^{+}, \boldsymbol{p}$ and $\boldsymbol{q}$, which can be written as,

$$
f\left(\boldsymbol{\pi}^{+}, \boldsymbol{\pi}^{-}, \boldsymbol{p}, \boldsymbol{q} \mid \boldsymbol{X}\right)=f\left(\boldsymbol{\pi}^{+}, \boldsymbol{\pi}^{-} \mid \hat{\boldsymbol{F}}^{1}, \hat{\boldsymbol{F}}^{0}, \boldsymbol{q}\right) f\left(\boldsymbol{q} \mid \boldsymbol{n}_{s}^{0}\right) f(\boldsymbol{p} \mid \boldsymbol{n})
$$

under Assumptions 1 and 2, where $\hat{\boldsymbol{F}}^{1}$ and $\hat{\boldsymbol{F}}^{0}$ are empirical CDFs corresponding to $\boldsymbol{F}^{1}$ and $\boldsymbol{F}^{0}$, respectively. For $\boldsymbol{p}$ and $\boldsymbol{q}$, we use the noninformative improper priors $\boldsymbol{p} \sim \operatorname{Dirichlet}(\mathbf{0})$ and $\boldsymbol{q}_{s} \sim$ $\operatorname{Dirichlet}(\mathbf{0}) \forall s \in \mathcal{A}$. Then, $\boldsymbol{q}_{s} \mid \boldsymbol{X} \sim \operatorname{Dirichlet}\left(\boldsymbol{n}_{s}^{0}\right) \forall s$ and $\boldsymbol{p} \mid \boldsymbol{X} \sim \operatorname{Dirichlet}(\boldsymbol{n})$.

We are now left with $f\left(\boldsymbol{\pi}^{+}, \boldsymbol{\pi}^{-} \mid \hat{\boldsymbol{F}}^{1}, \hat{\boldsymbol{F}}^{0}, \boldsymbol{q}\right)$. Because of the way these bounds are constructed (see Proposition [1],

$$
\begin{array}{llll}
\pi^{+}(a \mid s, c), \pi^{-}(a \mid s, c) & \Perp & \pi^{+}\left(a \mid s^{\prime}, c\right), \pi^{-}\left(a \mid s^{\prime}, c\right) \mid \hat{\boldsymbol{F}}^{1}, \hat{\boldsymbol{F}}^{0}, \boldsymbol{q} \text { and } \\
\pi^{+}(a \mid s, c), \pi^{-}(a \mid s, c) & \Perp & \pi^{+}\left(a^{\prime} \mid s, c\right), \pi^{-}\left(a^{\prime} \mid s, c\right) \mid \hat{\boldsymbol{F}}^{1}, \hat{\boldsymbol{F}}^{0}, \boldsymbol{q}
\end{array}
$$

for $s \neq s^{\prime}$ and $a \neq a^{\prime}$. Therefore, to fully characterize the posterior of $\left[\tau^{-}\left(a^{\prime}, a^{\prime \prime} \mid c\right), \tau^{+}\left(a^{\prime}, a^{\prime \prime} \mid c\right)\right]$ for each $a, a^{\prime \prime}$ and $c \in \mathcal{A}$, it is sufficient to only consider the bivariate posterior distribution of $\left[\pi^{+}(a \mid s, c), \pi^{-}(a \mid s, c)\right]$ for $a \in\left\{a^{\prime}, a^{\prime \prime}\right\}$ and $s \in \mathcal{A}$. Note that, under mild assumptions and with a sufficiently large sample size, the posterior for each pair $\left[\pi^{+}(a \mid s, c), \pi^{-}(a \mid s, c)\right]$ can be approximated by a bivariate normal distribution due to the Bayesian central limit theorem. That is, we have:

$$
\left[\begin{array}{c}
\pi^{-}(a \mid s, c) \\
\pi^{+}(a \mid s, c)
\end{array}\right] \mid \boldsymbol{q}, \boldsymbol{X} \approx \operatorname{Normal}\left(\left[\begin{array}{c}
\bar{\pi}^{-}\left(a \mid s, c, \boldsymbol{q}_{s}, \boldsymbol{X}\right) \\
\bar{\pi}^{+}\left(a \mid s, c, \boldsymbol{q}_{s}, \boldsymbol{X}\right)
\end{array}\right],\left[\begin{array}{cc}
V^{-}\left(a \mid s, c, \boldsymbol{q}_{s}, \boldsymbol{X}\right) & C\left(a \mid s, c, \boldsymbol{q}_{s}, \boldsymbol{X}\right) \\
C\left(a \mid s, c, \boldsymbol{q}_{s}, \boldsymbol{X}\right) & V^{+}\left(a \mid s, c, \boldsymbol{q}_{s}, \boldsymbol{X}\right)
\end{array}\right]\right)
$$

when $N$ is sufficiently large, and the means and covariances can be approximated by the asymptotic means and covariances of the frequentist sampling distributions of $\left[\pi^{-}(a \mid s, c), \pi^{+}(a \mid s, c)\right]$, respectively, as shown below. Note that priors on $\pi^{-}(a \mid s, c), \pi^{+}(a \mid s, c)$ can be ignored and therefore left unspecified 
when $N$ is large because of the Bernstein-von Mises theorem.

Let $\underline{y}$ be the natural lower bound of $Y_{i}(a)$ if it exists and $\min \left\{Y_{i}: S_{i}=s, A_{i}=a\right\}$, which is the lowest point at which the estimated conditional $\operatorname{CDF}, \hat{\Gamma}_{a}(y, \infty \mid s, a)$, is nonzero, if it does not. Let $\Gamma_{a}^{-1}(\cdot)$ be the inverse of $\Gamma_{a}(y, \infty \mid s, a)$ (see Section A.3 for the definition) with respect to $y$, so that $\Gamma_{a}^{-1}\left(\Gamma_{a}(y, \infty \mid s, a)\right)=y$, and let $\hat{\Gamma}_{a}^{-1}(\cdot)$ be its sample analogue, such that $\hat{\Gamma}_{a}^{-1}(p)=\min \{y: p \leq$ $\left.\hat{\Gamma}_{a}(y, \infty \mid s, a)\right\}$. Let $b=\frac{q_{s c}}{1-q_{s a}}$. For the means, note that the $\pi^{-}(a \mid s, c)$ and $\pi^{+}(a \mid s, c)$ are functions of $F(y \mid s, a, 0), F(y \mid s, a, 1)$ and $P(a \mid s, 0)$ (as shown in Appendix A.3, which can be consistently estimated by their nonparametric maximum likelihood estimates $\hat{F}(y \mid s, a, 0), \hat{F}(y \mid s, a, 1)$ and $q_{s a}$, respectively. This implies the following plug-in estimators for $\bar{\pi}^{-}\left(a \mid s, c, \boldsymbol{q}_{s}, \boldsymbol{X}\right)$ and $\bar{\pi}^{+}\left(a \mid s, c, \boldsymbol{q}_{s}, \boldsymbol{X}\right)$ :

$$
\begin{aligned}
& \hat{\bar{\pi}}^{-}\left(a \mid s, c, \boldsymbol{q}_{s}, \boldsymbol{X}\right)=\hat{\Gamma}_{a}^{-1}(b)-\int_{\underline{y}}^{\hat{\Gamma}_{a}^{-1}(b)} \frac{\hat{F}(y \mid s, a, 1)-\hat{F}(y \mid s, a, 0) q_{s a}}{q_{s c}} \mathrm{~d} y \\
& \hat{\bar{\pi}}^{+}\left(a \mid s, c, \boldsymbol{q}_{s}, \boldsymbol{X}\right)=\hat{\Gamma}_{a}^{-1}(1-b)-\int_{\hat{\Gamma}_{a}^{-1}(1-b)}^{\infty} \frac{q_{s a}+\hat{F}(y \mid s, a, 1)-\hat{F}(y \mid s, a, 0) q_{s a}-1}{q_{s c}} \mathrm{~d} y,
\end{aligned}
$$

where we used the fact that $\Phi_{a}^{+}(y \mid s, c)=1$ for $y \geq \Gamma_{a}^{-1}(b)$ and $\Phi_{a}^{-}(y \mid s, c)=0$ for $y \leq \Gamma_{a}^{-1}(1-b)$ (see Appendix A.3 for the definitions of $\Phi_{a}^{+}(y \mid s, c)$ and $\left.\Phi_{a}^{-}(y \mid s, c)\right)$.

For the variances and covariances, we use the fact that for any $\operatorname{ECDF} \hat{F}(\cdot), \operatorname{Cov}(\hat{F}(a), \hat{F}(b))=$ $\frac{F(a)-F(a) F(b)}{n}$ for $a \leq b$ where $n$ is the number of steps in $\hat{F}(\cdot)$.

$$
\begin{aligned}
& V^{-}\left(a \mid s, c, \boldsymbol{q}_{s}, \boldsymbol{X}\right) \\
& =\operatorname{Var}\left(\hat{\Gamma}_{a}^{-1}(b)-\int_{\underline{y}}^{\hat{\Gamma}_{a}^{-1}(b)} \frac{\hat{F}(y \mid s, a, 1)-\hat{F}(y \mid s, a, 0) q_{s a}}{q_{s c}} \mathrm{~d} y\right) \\
& =\left(\frac{1}{q_{s c}}\right)^{2} \operatorname{Var}\left(\int_{\underline{y}}^{\hat{\Gamma}_{a}^{-1}(b)} \hat{F}(y \mid s, a, 1)-\hat{F}(y \mid s, a, 0) q_{s a} \mathrm{~d} y\right) \\
& =\left(\frac{1}{q_{s c}}\right)^{2} \int_{\underline{y}}^{\hat{\Gamma}_{a}^{-1}(b)} \int_{\underline{y}}^{\hat{\Gamma}_{a}^{-1}(b)} \operatorname{Cov}\left(\begin{array}{c}
\hat{F}(y \mid s, a, 1)-\hat{F}(y \mid s, a, 0) q_{s a}, \\
\hat{F}(x \mid s, a, 1)-\hat{F}(x \mid s, a, 0) q_{s a}
\end{array}\right) \mathrm{d} x \mathrm{~d} y \\
& =2\left(\frac{1}{q_{s c}}\right)^{2} \int_{\underline{y}}^{\hat{\Gamma}_{a}^{-1}(b)} \int_{y}^{\hat{\Gamma}_{a}^{-1}(b)} \operatorname{Cov}\left(\begin{array}{c}
\hat{F}(y \mid s, a, 1)-\hat{F}(y \mid s, a, 0) q_{s a}, \\
\hat{F}(x \mid s, a, 1)-\hat{F}(x \mid s, a, 0) q_{s a}
\end{array}\right) \mathrm{d} x \mathrm{~d} y
\end{aligned}
$$




$$
\begin{aligned}
=2 & \left(\frac{1}{q_{s c}}\right)^{2} \int_{\underline{y}}^{\hat{\Gamma}_{a}^{-1}(b)} \int_{y}^{\hat{\Gamma}_{a}^{-1}(b)} \operatorname{Cov}(\hat{F}(y \mid s, a, 1), \hat{F}(x \mid s, a, 1)) \mathrm{d} x \mathrm{~d} y \\
& +2\left(\frac{q_{s a}}{q_{s c}}\right)^{2} \int_{\underline{y}}^{\hat{\Gamma}_{a}^{-1}(b)} \int_{y}^{\hat{\Gamma}_{a}^{-1}(b)} \operatorname{Cov}(\hat{F}(y \mid s, a, 0), \hat{F}(x \mid s, a, 0)) \mathrm{d} x \mathrm{~d} y \\
= & \frac{2}{n_{s a}^{1}}\left(\frac{1}{q_{s c}}\right)^{2} \int_{\underline{y}}^{\hat{\Gamma}_{a}^{-1}(b)} \int_{y}^{\hat{\Gamma}_{a}^{-1}(b)} F(y \mid s, a, 1)(1-F(x \mid s, a, 1)) \mathrm{d} x \mathrm{~d} y \\
& +\frac{2}{n_{s a}^{0}}\left(\frac{q_{s a}}{q_{s c}}\right)^{2} \int_{\underline{y}}^{\hat{\Gamma}_{a}^{-1}(b)} \int_{y}^{\hat{\Gamma}_{a}^{-1}(b)} F(y \mid s, a, 0)(1-F(x \mid s, a, 0)) \mathrm{d} x \mathrm{~d} y,
\end{aligned}
$$

where $n_{s a}^{0}$ is as defined in Section 6 and $n_{s a}^{1}=\sum_{i=1}^{N} \mathbf{1}\left\{S_{i}=s, A_{i}=a, D_{i}=1\right\}$. Similarly,

$$
\begin{aligned}
V^{+}\left(a \mid s, c, \boldsymbol{q}_{s}, \boldsymbol{X}\right) \\
=\operatorname{Var}\left(\hat{\Gamma}_{a}^{-1}(1-b)-\int_{\hat{\Gamma}_{a}^{-1}(1-b)}^{\infty} \frac{q_{s a}+\hat{F}(y \mid s, a, 1)-\hat{F}(y \mid s, a, 0) q_{s a}-1}{q_{s c}} \mathrm{~d} y\right) \\
=\frac{2}{n_{s a}^{1}}\left(\frac{1}{q_{s c}}\right)^{2} \int_{\hat{\Gamma}_{a}^{-1}(1-b)}^{\infty} \int_{y}^{\infty} F(y \mid s, a, 1)(1-F(x \mid s, a, 1)) \mathrm{d} x \mathrm{~d} y \\
\quad+\frac{2}{n_{s a}^{0}}\left(\frac{q_{s a}}{q_{s c}}\right)^{2} \int_{\hat{\Gamma}_{a}^{-1}(1-b)}^{\infty} \int_{y}^{\infty} F(y \mid s, a, 0)(1-F(x \mid s, a, 0)) \mathrm{d} x \mathrm{~d} y
\end{aligned}
$$

We estimate these quantities by substituting $F(\cdot \mid s, a, d)$ with $\hat{F}(\cdot \mid s, a, d)$ for $d=0,1$. A small sample correction can optionally be applied to these estimates by replacing $n_{s a}^{d}$ with $n_{s a}^{d}-1$ for $d=0,1$.

The covariance between $\pi^{-}(a \mid s, c)$ and $\pi^{+}(a \mid s, c)$ depends on whether $b<\frac{1}{2}$, in which case they are based on disjoint (but still correlated) portions of the same ECDFs, or whether $b \geq \frac{1}{2}$, in which case they are based on overlapping regions of the ECDFs and are therefore more correlated. If $b \geq \frac{1}{2}$,

$$
\begin{aligned}
& C\left(a \mid s, c, \boldsymbol{q}_{s}, \boldsymbol{X}\right) \\
&=\operatorname{Cov}\left(\hat{\Gamma}_{a}^{-1}(b)-\int_{\underline{y}}^{\hat{\Gamma}_{a}^{-1}(b)} \frac{\hat{F}(y \mid s, a, 1)-\hat{F}(y \mid s, a, 0) q_{s a}}{q_{s c}} \mathrm{~d} y\right. \\
&\left.\quad \hat{\Gamma}_{a}^{-1}(1-b)-\int_{\hat{\Gamma}_{a}^{-1}(1-b)}^{\infty} \frac{q_{s a}+\hat{F}(y \mid s, a, 1)-\hat{F}(y \mid s, a, 0) q_{s a}-1}{q_{s c}} \mathrm{~d} y\right) \\
&=\operatorname{Cov}\left(\int_{\underline{y}}^{\hat{\Gamma}_{a}^{-1}(b)} \frac{\hat{F}(y \mid s, a, 1)-\hat{F}(y \mid s, a, 0) q_{s a}}{q_{s c}} \mathrm{~d} y, \int_{\hat{\Gamma}_{a}^{-1}(1-b)}^{\infty} \frac{\hat{F}(y \mid s, a, 1)-\hat{F}(y \mid s, a, 0) q_{s a}}{q_{s c}}\right)
\end{aligned}
$$




$$
\begin{aligned}
& =\left(\frac{1}{q_{s c}}\right)^{2} \int_{\underline{y}}^{\hat{\Gamma}_{a}^{-1}(1-b)} \int_{\hat{\Gamma}_{a}^{-1}(1-b)}^{\infty} \operatorname{Cov}\left(\begin{array}{c}
\hat{F}(y \mid s, a, 1)-\hat{F}(y \mid s, a, 0) q_{s a}, \\
\hat{F}(x \mid s, a, 1)-\hat{F}(x \mid s, a, 0) q_{s a}
\end{array}\right) \mathrm{d} x \mathrm{~d} y \\
& +2\left(\frac{1}{q_{s c}}\right)^{2} \int_{\hat{\Gamma}_{a}^{-1}(1-b)}^{\hat{\Gamma}_{a}^{-1}(b)} \int_{y}^{\hat{\Gamma}_{a}^{-1}(b)} \operatorname{Cov}\left(\begin{array}{c}
\hat{F}(y \mid s, a, 1)-\hat{F}(y \mid s, a, 0) q_{s a}, \\
\hat{F}(x \mid s, a, 1)-\hat{F}(x \mid s, a, 0) q_{s a}
\end{array}\right) \mathrm{d} x \mathrm{~d} y \\
& +\left(\frac{1}{q_{s c}}\right)^{2} \int_{\hat{\Gamma}_{a}^{-1}(1-b)}^{\hat{\Gamma}_{a}^{-1}(b)} \int_{\hat{\Gamma}_{a}^{-1}(b)}^{\infty} \operatorname{Cov}\left(\begin{array}{c}
\hat{F}(y \mid s, a, 1)-\hat{F}(y \mid s, a, 0) q_{s a}, \\
\hat{F}(x \mid s, a, 1)-\hat{F}(x \mid s, a, 0) q_{s a}
\end{array}\right) \mathrm{d} x \mathrm{~d} y \\
& =\frac{1}{n_{s a}^{1}}\left(\frac{1}{q_{s c}}\right)^{2} \int_{\underline{y}}^{\hat{\Gamma}_{a}^{-1}(1-b)} \int_{\hat{\Gamma}_{a}^{-1}(1-b)}^{\infty} F(y \mid s, a, 1)(1-F(x \mid s, a, 1)) \mathrm{d} x \mathrm{~d} y \\
& +\frac{1}{n_{s a}^{0}}\left(\frac{q_{s a}}{q_{s c}}\right)^{2} \int_{\underline{y}}^{\hat{\Gamma}_{a}^{-1}(1-b)} \int_{\hat{\Gamma}_{a}^{-1}(1-b)}^{\infty} F(y \mid s, a, 0)(1-F(x \mid s, a, 0)) \mathrm{d} x \mathrm{~d} y \\
& +\frac{2}{n_{s a}^{1}}\left(\frac{1}{q_{s c}}\right)^{2} \int_{\hat{\Gamma}_{a}^{-1}(1-b)}^{\hat{\Gamma}_{a}^{-1}(b)} \int_{y}^{\hat{\Gamma}_{a}^{-1}(b)} F(y \mid s, a, 1)(1-F(x \mid s, a, 1)) \mathrm{d} x \mathrm{~d} y \\
& +\frac{2}{n_{s a}^{0}}\left(\frac{q_{s a}}{q_{s c}}\right)^{2} \int_{\hat{\Gamma}_{a}^{-1}(1-b)}^{\hat{\Gamma}_{a}^{-1}(b)} \int_{y}^{\hat{\Gamma}_{a}^{-1}(b)} F(y \mid s, a, 0)(1-F(x \mid s, a, 0)) \mathrm{d} x \mathrm{~d} y \\
& +\frac{1}{n_{s a}^{1}}\left(\frac{1}{q_{s c}}\right)^{2} \int_{\hat{\Gamma}_{a}^{-1}(1-b)}^{\hat{\Gamma}_{a}^{-1}(b)} \int_{\hat{\Gamma}_{a}^{-1}(b)}^{\infty} F(y \mid s, a, 1)(1-F(x \mid s, a, 1)) \mathrm{d} x \mathrm{~d} y \\
& +\frac{1}{n_{s a}^{0}}\left(\frac{q_{s a}}{q_{s c}}\right)^{2} \int_{\hat{\Gamma}_{a}^{-1}(1-b)}^{\hat{\Gamma}_{a}^{-1}(b)} \int_{\hat{\Gamma}_{a}^{-1}(b)}^{\infty} F(y \mid s, a, 0)(1-F(x \mid s, a, 0)) \mathrm{d} x \mathrm{~d} y
\end{aligned}
$$

and if $b<\frac{1}{2}$,

$$
\begin{aligned}
C\left(a \mid s, c, \boldsymbol{q}_{s}, \boldsymbol{X}\right) & \\
= & \frac{1}{n_{s a}^{1}}\left(\frac{1}{q_{s c}}\right)^{2} \int_{\underline{y}}^{\hat{\Gamma}_{a}^{-1}(b)} \int_{\hat{\Gamma}_{a}^{-1}(1-b)}^{\infty} F(y \mid s, a, 1)(1-F(x \mid s, a, 1)) \mathrm{d} x \mathrm{~d} y \\
& \quad+\frac{1}{n_{s a}^{0}}\left(\frac{q_{s a}}{q_{s c}}\right)^{2} \int_{\underline{y}}^{\hat{\Gamma}_{a}^{-1}(b)} \int_{\hat{\Gamma}_{a}^{-1}(1-b)}^{\infty} F(y \mid s, a, 0)(1-F(x \mid s, a, 0)) \mathrm{d} x \mathrm{~d} y .
\end{aligned}
$$

Again, we estimate these by replacing $F(\cdot \mid s, a, d)$ with $\hat{F}(\cdot \mid s, a, d)$ for $d=0,1$. The small sample correction can also be applied.

Finally, in the special case of $a=c$, the quantity $\pi(a \mid s, c)=\pi(c \mid s, c)$ is point-identified. Therefore, equation (23) reduces to a univariate normal distribution such that $\bar{\pi} \equiv \bar{\pi}^{-}\left(c \mid s, c, \boldsymbol{q}_{s}, \boldsymbol{X}\right)=$ $\bar{\pi}^{+}\left(c \mid s, c, \boldsymbol{q}_{s}, \boldsymbol{X}\right)$ and $V \equiv V^{-}\left(c \mid s, c, \boldsymbol{q}_{s}, \boldsymbol{X}\right)=V^{+}\left(c \mid s, c, \boldsymbol{q}_{s}, \boldsymbol{X}\right)=C\left(c \mid s, c, \boldsymbol{q}_{s}, \boldsymbol{X}\right)$. In fact, the es- 
timators of these parameters provided above reduce to the sample mean and the sampling variance for the mean, respectively, for the corresponding subgroup:

$$
\begin{aligned}
\hat{\bar{\pi}} & =\underline{y}+\int_{\underline{y}}^{\infty} 1-\hat{F}(y \mid s, c, 0) \mathrm{d} y \\
& =\underline{y}+\int_{\underline{y}}^{\infty} \sum_{i=1}^{N}\left(1-\mathbf{1}\left\{Y_{i} \leq y\right\}\right) \cdot \frac{\mathbf{1}\left\{S_{i}=s, A_{i}=c, D_{i}=0\right\}}{n_{s c}^{0}} \mathrm{~d} y \\
& =\underline{y}+\frac{1}{n_{s c}^{0}} \sum_{i=1}^{N}\left(\int_{\underline{y}}^{Y_{i}} 1 \mathrm{~d} y+\int_{Y_{i}}^{\infty} 0 \mathrm{~d} y\right) \cdot \mathbf{1}\left\{S_{i}=s, A_{i}=c, D_{i}=0\right\} \\
& =\frac{1}{n_{s c}^{0}} \sum_{i=1}^{N} Y_{i} \cdot \mathbf{1}\left\{S_{i}=s, A_{i}=c, D_{i}=0\right\},
\end{aligned}
$$

and

$$
\begin{aligned}
\hat{V}= & \frac{2}{n_{s c}^{0}} \int_{\underline{y}}^{\infty} \int_{y}^{\infty} \hat{F}(y \mid s, c, 0)(1-\hat{F}(x \mid s, c, 0)) \mathrm{d} x \mathrm{~d} y \\
= & \frac{2}{n_{s c}^{0}} \int_{\underline{y}}^{\infty} \int_{y}^{\infty}\left(\sum_{i=1}^{N} \mathbf{1}\left\{Y_{i} \leq y\right\} \cdot \frac{\mathbf{1}\left\{S_{i}=s, A_{i}=c, D_{i}=0\right\}}{n_{s c}^{0}}\right) \\
& \times\left(\sum_{j=1}^{N}\left(1-\mathbf{1}\left\{Y_{j} \leq x\right\}\right) \cdot \frac{\mathbf{1}\left\{S_{j}=s, A_{j}=c, D_{j}=0\right\}}{n_{s c}^{0}}\right) \mathrm{d} x \mathrm{~d} y \\
= & \frac{2}{\left(n_{s c}^{0}\right)^{3}} \int_{\underline{y}}^{\infty}\left(\sum_{i=1}^{N} \mathbf{1}\left\{Y_{i} \leq y\right\} \cdot \mathbf{1}\left\{S_{i}=s, A_{i}=c, D_{i}=0\right\}\right) \\
= & \frac{2}{\left(n_{s c}^{0}\right)^{3}} \sum_{i=1}^{N} \sum_{j=1}^{N} \int_{\underline{y}}^{\infty} \mathbf{1}\left\{Y_{i} \leq y\right\} \cdot \mathbf{1}\left\{S_{i}=s, A_{i}=c, D_{i}=0\right\} \\
= & \left.\left.\frac{1}{\left(n_{s c}^{0}\right)^{2}} \sum_{i=1}^{N}\left(Y_{i}-\bar{\pi}\right)^{2} \cdot \mathbf{1}\left\{S_{i}=s, A_{i}=c, D_{i}=0\right\}, \mathbf{1}\left\{Y_{j} \leq x\right\}\right) \cdot \mathbf{1}\left\{S_{j}=s, A_{j}=c, D_{j}=0\right\} \mathrm{d} x\right) \mathrm{d} y \\
= & \frac{1}{\left(n_{s c}^{0}\right)^{3}} \sum_{i=1}^{N} \sum_{j \in \mathcal{J}}^{N} \sum_{j \in \mathcal{J}} \frac{\left(Y_{j}-Y_{i}\right)^{2}}{\left(n_{s c}^{0}\right)^{2}} \cdot \mathbf{1}\left\{S_{i}=s, A_{i}=c, D_{i}=0\right\} \cdot \mathbf{1}\left\{S_{j}=s, A_{j}=c, D_{j}=0\right\} \\
\text { with } \mathcal{J}=\left\{j \in 1, \cdots, N: Y_{j} \geq Y_{i}\right\} & \left.\mathbf{1}\left\{Y_{j} \leq y\right\}\right)\left(Y_{j}-y\right) \cdot \mathbf{1}\left\{S_{j}=s, A_{j}=c, D_{j}=0\right\} \mathrm{d} y \\
Y_{j} & \left(Y_{j}-y\right) \mathrm{d} y
\end{aligned}
$$


for any $c, s \in \mathcal{A}$. Again, a small sample correction can be applied for $\hat{V}$ by multiplying it by $n_{s c}^{0} /\left(n_{s c}^{0}-1\right)$.

For the binary-outcome bounds in Proposition 2, we employ a similar procedure. Let $\boldsymbol{H}=\left[H_{s a}\right]=$ $\left[\operatorname{Pr}\left(Y_{i}=1 \mid S_{i}=s, A_{i}=a, D_{i}=1\right)\right]$ and $\boldsymbol{G}=\left[G_{s a}\right]=\left[\operatorname{Pr}\left(Y_{i}=1 \mid S_{i}=s, A_{i}=a, D_{i}=0\right)\right]$. In this case, $\boldsymbol{\tau}^{-}$and $\boldsymbol{\tau}^{+}$are completely determined by $\boldsymbol{H}, \boldsymbol{G}, \boldsymbol{p}$ and $\boldsymbol{q}$. The endpoints of the ACTE bounds $\tau^{-}\left(a, a^{\prime} \mid c\right)$ and $\tau^{+}\left(a, a^{\prime} \mid c\right)$ are respectively given by the solutions to the linear problem described in Proposition 2 .

$$
\min _{\Phi} \text { and } \max _{\Phi} \frac{1}{\operatorname{Pr}\left(A_{i}=c \mid D_{i}=0\right)}\left\{\sum_{a^{\prime \prime} \in\{0,1\}} \sum_{s \in \mathcal{A}}\left(\phi_{1,0, y_{a^{\prime \prime}}, s, c}-\phi_{0,1, y_{a^{\prime \prime}}, s, c}\right)\right\}
$$

s.t. $\quad \phi_{y_{0}, y_{1}, y_{2}, s, c^{\prime}} \geq 0 \forall y_{0}, y_{1}, y_{2}, s, c^{\prime}, \sum_{y_{0} \in\{0,1\}} \sum_{y_{1} \in\{0,1\}} \sum_{y_{2} \in\{0,1\}} \sum_{s \in \mathcal{A}} \sum_{c^{\prime} \in \mathcal{A}} \phi_{y_{0}, y_{1}, y_{2}, s, c^{\prime}}=1$, $\sum_{y_{0} \in\{0,1\}} \sum_{y_{1} \in\{0,1\}} \sum_{y_{2} \in\{0,1\}} \phi_{y_{0}, y_{1}, y_{2}, s, c^{\prime}} \cdot \mathbf{1}\left\{y_{c^{\prime}}=1\right\}=q_{s c^{\prime}} p_{s} G_{s c^{\prime}} \forall s, c^{\prime}, \sum_{y_{0} \in\{0,1\}} \sum_{y_{1} \in\{0,1\}} \sum_{y_{2} \in\{0,1\}}$ $\phi_{y_{0}, y_{1}, y_{2}, s, c^{\prime}}=q_{s c^{\prime}} p_{s} \forall s, c^{\prime}$, and $\sum_{y_{0} \in\{0,1\}} \sum_{y_{1} \in\{0,1\}} \sum_{y_{2} \in\{0,1\}} \sum_{c^{\prime} \in \mathcal{A}} \phi_{y_{0}, y_{1}, y_{2}, s, c^{\prime}} \cdot \mathbf{1}\left\{y_{a^{\prime \prime}}=1\right\}=p_{s} H_{s a^{\prime \prime}}$ $\forall s, a^{\prime \prime}$, where $\Phi \equiv\left\{\phi_{y_{0}, y_{1}, y_{2}, s, c}: y_{0} \in\{0,1\}, y_{1} \in\{0,1\}, y_{2} \in\{0,1\}, s \in \mathcal{A}, c \in \mathcal{A}\right\}$.

The joint posterior of these parameters can be factorized as $f(\boldsymbol{H}, \boldsymbol{G}, \boldsymbol{p}, \boldsymbol{q} \mid \boldsymbol{X})=f\left(\boldsymbol{H} \mid \hat{\boldsymbol{F}}^{1}\right) f\left(\boldsymbol{G} \mid \hat{\boldsymbol{F}}^{0}\right)$ $f\left(\boldsymbol{q} \mid \boldsymbol{n}_{s}^{0}\right) f(\boldsymbol{p} \mid \boldsymbol{n})$ under Assumptions 1 and 2 . We use the improper priors $H_{s a} \sim \operatorname{Beta}(0,0)$ and $G_{s a} \sim$ $\operatorname{Beta}(0,0)$. The posteriors are then given by $H_{s a} \sim \operatorname{Beta}\left(\sum_{i=1}^{N} \mathbf{1}\left\{Y_{i}=1, S_{i}=s, A_{i}=a, D_{i}=\right.\right.$ $\left.1\}, \sum_{i=1}^{N} \mathbf{1}\left\{Y_{i}=0, S_{i}=s, A_{i}=a, D_{i}=1\right\}\right)$ and $G_{s a} \sim \operatorname{Beta}\left(\sum_{i=1}^{N} \mathbf{1}\left\{Y_{i}=1, S_{i}=s, A_{i}=a, D_{i}=\right.\right.$ $\left.0\}, \sum_{i=1}^{N} \mathbf{1}\left\{Y_{i}=0, S_{i}=s, A_{i}=a, D_{i}=0\right\}\right)$.

\section{A.6 Statistical Inference for the Sensitivity Analysis}

Our approach to statistical inference for the sensitivity analysis in Section 5 is similar to the procedure outlined in Section 6. In addition to the parameters defined there, we have the naïve estimates $\boldsymbol{\eta}=$ $\{\eta(a \mid s): a, s \in \mathcal{A}\}$, where $\eta(a \mid s)=\mathbb{E}\left[Y_{i} \mid S_{i}=s, A_{i}=a, D_{i}=1\right]$. For a given value of the sensitivity parameter, $\rho=\rho_{a c}=\rho_{a^{\prime} c}$, the sets of upper and lower bounds on $\tau\left(a, a^{\prime} \mid c\right)$ are denoted $\tau_{\rho}^{-}$and $\tau_{\rho}^{+}$for $a, a^{\prime}, c \in \mathcal{A}$.

Given $\boldsymbol{\pi}^{-}, \boldsymbol{\pi}^{+}, \boldsymbol{p}, \boldsymbol{q}$, and $\boldsymbol{\eta}$, we can deterministically find $\boldsymbol{\tau}_{\rho}^{-}$and $\boldsymbol{\tau}_{\rho}^{+}$. Each pair of $\tau_{\rho}^{-}\left(a, a^{\prime} \mid c\right)$ and 
$\tau_{\rho}^{+}\left(a, a^{\prime} \mid c\right)$ are equal to the endpoints of the following interval:

$$
\begin{aligned}
& \tau\left(a, a^{\prime} \mid c\right) \in \\
& \left(\left[\eta(a \mid c)-\rho_{a c}, \eta(a \mid c)+\rho_{a c}\right] \bigcap\left[\sum_{s \in \mathcal{A}} \pi^{-}(a \mid s, c) \frac{q_{s c} p_{s}}{\sum_{s^{\prime} \in \mathcal{A}} q_{s^{\prime} c} p_{s^{\prime}}}, \sum_{s \in \mathcal{A}} \pi^{+}(a \mid s, c) \frac{q_{s c} p_{s}}{\sum_{s^{\prime} \in \mathcal{A}} q_{s^{\prime} c} p_{s^{\prime}}}\right]\right) \\
& \quad-\left(\left[\eta(a \mid c)-\rho_{a^{\prime} c}, \eta(a \mid c)+\rho_{a c}\right] \bigcap\left[\sum_{s \in \mathcal{A}} \pi^{-}\left(a^{\prime} \mid s, c\right) \frac{q_{s c} p_{s}}{\sum_{s^{\prime} \in \mathcal{A}} q_{s^{\prime} c} p_{s^{\prime}}}, \sum_{s \in \mathcal{A}} \pi^{+}\left(a^{\prime} \mid s, c\right) \frac{q_{s c} p_{s}}{\sum_{s^{\prime} \in \mathcal{A}} q_{s^{\prime} c} p_{s^{\prime}}}\right]\right)
\end{aligned}
$$

We therefore simulate from the posterior of $\left(\boldsymbol{\tau}^{-}, \boldsymbol{\tau}^{+}\right)$by drawing samples of $\boldsymbol{\pi}^{-}, \boldsymbol{\pi}^{+}, \boldsymbol{\eta}, \boldsymbol{p}$ and $\boldsymbol{q}$,

$$
f\left(\boldsymbol{\pi}^{+}, \boldsymbol{\pi}^{-}, \boldsymbol{\eta}, \boldsymbol{p}, \boldsymbol{q} \mid \boldsymbol{X}\right)=f\left(\boldsymbol{\pi}^{+}, \boldsymbol{\pi}^{-}, \boldsymbol{\eta} \mid \hat{\boldsymbol{F}}^{1}, \hat{\boldsymbol{F}}^{0}, \boldsymbol{q}\right) f\left(\boldsymbol{q} \mid \boldsymbol{n}_{s}^{0}\right) f(\boldsymbol{p} \mid \boldsymbol{n})
$$

under Assumptions 1 and 2. Note that this differs from Appendix A.5 only in that the distributions of $\pi^{+}$and $\pi^{-}$are considered jointly with $\boldsymbol{\eta}$. These have the additional independence relations

$$
\begin{array}{lll}
\eta(a \mid s) & \Perp & \pi^{+}\left(a \mid s^{\prime}, c\right), \pi^{-}\left(a \mid s^{\prime}, c\right), \eta\left(a \mid s^{\prime}\right) \mid \hat{\boldsymbol{F}}^{1}, \hat{\boldsymbol{F}}^{0}, \boldsymbol{q} \text { and } \\
\eta(a \mid s) & \Perp & \pi^{+}\left(a^{\prime} \mid s, c\right), \pi^{-}\left(a^{\prime} \mid s, c\right), \eta\left(a^{\prime} \mid s\right) \mid \hat{\boldsymbol{F}}^{1}, \hat{\boldsymbol{F}}^{0}, \boldsymbol{q}
\end{array}
$$

for $s \neq s^{\prime}$ and $a \neq a^{\prime}$.

We can therefore approximate the posterior of sensitivity bounds by Monte Carlo simulation of $\boldsymbol{p}$, $\boldsymbol{q}$, and the trivariate distributions $\left[\pi^{-}(a \mid s, c), \pi^{+}(a \mid s, c), \eta(a \mid c)\right]$ for $a \in\left\{a^{\prime}, a^{\prime \prime}\right\}$ and $s \in \mathcal{A}$. By the Bayesian central limit theorem, the latter is given by

$$
\begin{gathered}
{\left[\begin{array}{c}
\pi^{-}(a \mid s, c) \\
\pi^{+}(a \mid s, c) \\
\eta(a \mid c)
\end{array}\right] \mid \boldsymbol{q}, \boldsymbol{X} \approx \text { Normal }\left(\left[\begin{array}{c}
\bar{\pi}^{-}\left(a \mid s, c, \boldsymbol{q}_{s}, \boldsymbol{X}\right) \\
\bar{\pi}^{+}\left(a \mid s, c, \boldsymbol{q}_{s}, \boldsymbol{X}\right) \\
\bar{\eta}(a \mid c, \boldsymbol{X})
\end{array}\right], \boldsymbol{\Sigma}(a \mid s, c)\right), \quad \text { where }} \\
\boldsymbol{\Sigma}(a \mid s, c)=\left[\begin{array}{ccc}
V^{-}\left(a \mid s, c, \boldsymbol{q}_{s}, \boldsymbol{X}\right) & C\left(a \mid s, c, \boldsymbol{q}_{s}, \boldsymbol{X}\right) & C_{\eta}^{-}\left(a \mid s, c, \boldsymbol{q}_{s}, \boldsymbol{X}\right) \\
C\left(a \mid s, c, \boldsymbol{q}_{s}, \boldsymbol{X}\right) & V^{+}\left(a \mid s, c, \boldsymbol{q}_{s}, \boldsymbol{X}\right) & C_{\eta}^{+}\left(a \mid s, c, \boldsymbol{q}_{s}, \boldsymbol{X}\right) \\
C_{\eta}^{-}\left(a \mid s, c, \boldsymbol{q}_{s}, \boldsymbol{X}\right) & C_{\eta}^{+}\left(a \mid s, c, \boldsymbol{q}_{s}, \boldsymbol{X}\right) & V_{\eta}\left(a \mid s, \boldsymbol{q}_{s}, \boldsymbol{X}\right)
\end{array}\right]
\end{gathered}
$$

when $N$ is large, and the additional parameters $\bar{\eta}, C_{\eta}^{-}, C_{\eta}^{+}$, and $V_{\eta}$ are defined below. 
Note that naïve estimate $\eta(a \mid s)$ is point-identified, and its posterior mean and variance are equivalent to the sample mean and the sampling variance for the mean for the corresponding forced-choice units. These are given by:

$$
\begin{aligned}
\bar{\eta}(a \mid s) & =\underline{y}+\int_{\underline{y}}^{\infty} 1-F(y \mid s, a, 1) \mathrm{d} y \\
V_{\eta}(a \mid s) & =\frac{2}{n_{s a}^{1}} \int_{\underline{y}}^{\infty} \int_{y}^{\infty} F(y \mid s, a, 1)(1-F(x \mid s, a, 1)) \mathrm{d} x \mathrm{~d} y .
\end{aligned}
$$

Derivations closely follow Section A.5 and therefore are omitted here. Estimation can be done by plug-in with an optional small sample correction.

The posterior of $\eta(a \mid s)$ covaries with those of $\pi^{-}(a \mid s, c)$ and $\pi^{+}(a \mid s, c)$ because the latter parameters depend partially on the ECDF of the same forced-choice units.

$$
\begin{aligned}
& C_{\eta}^{-}\left(a \mid s, c, \boldsymbol{q}_{s}, \boldsymbol{X}\right) \\
& =\operatorname{Cov}\left(\hat{\Gamma}_{a}^{-1}(b)-\int_{\underline{y}}^{\hat{\Gamma}_{a}^{-1}(b)} \frac{\hat{F}(y \mid s, a, 1)-\hat{F}(y \mid s, a, 0) q_{s a}}{q_{s c}} \mathrm{~d} y, \underline{y}+\int_{\underline{y}}^{\infty} 1-\hat{F}(y \mid s, a, 1) \mathrm{d} y\right) \\
& =\frac{2}{n_{s a}^{1} \cdot q_{s c}} \int_{\underline{y}}^{\hat{\Gamma}_{a}^{-1}(b)} \int_{y}^{\hat{\Gamma}_{a}^{-1}(b)} F(y \mid s, a, 1)(1-F(x \mid s, a, 1)) \mathrm{d} x \mathrm{~d} y \\
& +\frac{1}{n_{s a}^{1} \cdot q_{s c}} \int_{\underline{y}}^{\hat{\Gamma}_{a}^{-1}(b)} \int_{\hat{\Gamma}_{a}^{-1}(b)}^{\infty} F(y \mid s, a, 1)(1-F(x \mid s, a, 1)) \mathrm{d} x \mathrm{~d} y \\
& C_{\eta}^{+}\left(a \mid s, c, \boldsymbol{q}_{s}, \boldsymbol{X}\right) \\
& =\operatorname{Cov}\left(\hat{\Gamma}_{a}^{-1}(1-b)-\int_{\hat{\Gamma}_{a}^{-1}(1-b)}^{\infty} \frac{q_{s a}+\hat{F}(y \mid s, a, 1)-\hat{F}(y \mid s, a, 0) q_{s a}-1}{q_{s c}} \mathrm{~d} y,\right. \\
& \left.\underline{y}+\int_{\underline{y}}^{\infty} 1-\hat{F}(y \mid s, a, 1) \mathrm{d} y\right) \\
& =\frac{1}{n_{s a}^{1} \cdot q_{s c}} \int_{\underline{y}}^{\hat{\Gamma}_{a}^{-1}(1-b)} \int_{\hat{\Gamma}_{a}^{-1}(1-b)}^{\infty} F(y \mid s, a, 1)(1-F(x \mid s, a, 1)) \mathrm{d} x \mathrm{~d} y \\
& +\frac{2}{n_{s a}^{1} \cdot q_{s c}} \int_{\hat{\Gamma}_{a}^{-1}(1-b)}^{\infty} \int_{y}^{\infty} F(y \mid s, a, 1)(1-F(x \mid s, a, 1)) \mathrm{d} x \mathrm{~d} y
\end{aligned}
$$

for any $s, c \neq a \in \mathcal{A}$.

Thus, each draw of the sensitivity results from their posterior is generated by the following procedure:

1. Draw $\boldsymbol{p} \equiv\left[p_{s}\right] \sim \operatorname{Dirichlet}(\boldsymbol{n})$, where $\boldsymbol{n} \equiv\left[n_{s}\right]=\left[\sum_{i=1}^{N} \mathbf{1}\left\{S_{i}=0\right\}, \cdots, \sum_{i=1}^{N} \mathbf{1}\left\{S_{i}=J-1\right\}\right]^{\top}$. 
2. For each $s \in \mathcal{A}$ :

(a) Draw $\boldsymbol{q}_{s} \equiv\left[q_{s a}\right] \sim \operatorname{Dirichlet}\left(\boldsymbol{n}_{s}^{0}\right)$, where $\boldsymbol{n}_{s}^{0} \equiv\left[n_{s a}^{0}\right]=\left[\sum_{i=1}^{N} \mathbf{1}\left\{S_{i}=s, A_{i}=0, D_{i}=\right.\right.$ $\left.0\}, \cdots, \sum_{i=1}^{N} \mathbf{1}\left\{S_{i}=s, A_{i}=J-1, D_{i}=0\right\}\right]^{\top} ;$

(b) For each $a$ and $c \in \mathcal{A}$, draw a triplet $\left[\pi^{-}(a \mid s, c), \pi^{+}(a \mid s, c), \eta(a \mid s)\right]$ from the trivariate normal distribution defined above.

3. For a given $\rho$, calculate a simulated draw of $\left[\tau_{\rho}^{-}\left(a, a^{\prime} \mid c\right), \tau_{\rho}^{+}\left(a, a^{\prime} \mid c\right)\right]$ according to equation (9).

The sensitivity procedure for binary outcomes differs only in the last two steps:

2. (b) For each $a \in \mathcal{A}$, draw $H_{s a}$ and $G_{s a}$ from the posteriors discussed in Sections 6 and A.5.

3. Calculate a simulated draw of $\left[\tau^{-}\left(a, a^{\prime} \mid c\right), \tau^{+}\left(a, a^{\prime} \mid c\right)\right]$ by solving the linear programming problem in equation (24), with the additional sensitivity constraints $\sum_{y_{0} \in\{0,1\}} \sum_{y_{1} \in\{0,1\}} \sum_{y_{2} \in\{0,1\}} \sum_{s \in \mathcal{A}}$ $\phi_{y_{0}, y_{1}, y_{2}, s, c} \mathbf{1}\left\{y_{a^{*}}=1\right\} \geq\left(H_{s a^{*}}-\rho_{a^{*} c}\right) \sum_{s \in \mathcal{A}} q_{s c} p_{s}$ and $\sum_{y_{0} \in\{0,1\}} \sum_{y_{1} \in\{0,1\}} \sum_{y_{2} \in\{0,1\}} \sum_{s \in \mathcal{A}} \phi_{y_{0}, y_{1}, y_{2}, s, c}$ $\mathbf{1}\left\{y_{a^{*}}=1\right\} \leq\left(H_{s a^{*}}+\rho_{a^{*} c}\right) \sum_{s \in \mathcal{A}} q_{s c} p_{s}$ for given $c$ and $a^{*} \in\left\{a, a^{\prime}\right\}$.

\section{A.7 Additional Simulation Results}

In this section, we present additional results from the simulations described in Section 8 . First, we explore the performance of the EM-algorithm-based parametric approach proposed by Long et al. (2008) (hereafter LLL) in a setting close to our empirical application. This necessitates extending LLL's original methodology, as it was developed for a binary treatment. We thus modify their parametric model to accommodate a categorical treatment by modeling the treatment choice with the multinomial logit model, as opposed to the binary logit model. (We have confirmed that our own R implementation of this extension replicates the simulation results reported by LLL in their original article almost exactly.) To make LLL's approach comparable to our proposed method in terms of observed information used, we set subjects' stated preferences as the covariate in their choice and outcome models (i.e., $X_{1 i}=X_{2 i}=S_{i}$ using their notation). We then apply the LLL estimator to the same 500 simulated datasets as in Section 8 . 


\begin{tabular}{rrrrr}
\hline & $\mathrm{CD}=0.00$ & $\mathrm{CD}=0.33$ & $\mathrm{CD}=0.67$ & $\mathrm{CD}=1.00$ \\
\hline LLL & 0.053 & 0.028 & 0.019 & -0.020 \\
naïve & 0.002 & 0.011 & 0.023 & 0.038 \\
min & -0.001 & -0.001 & -0.001 & 0.000 \\
$\max$ & -0.001 & -0.001 & -0.001 & -0.001 \\
\hline
\end{tabular}

Table A.1: LLL bias for various CD values, holding OD at zero. Naïve and bounds biases from Section 8.1 are reproduced here for convenience.

\begin{tabular}{rrrrr}
\hline & $\mathrm{OD}=0.00$ & $\mathrm{OD}=0.33$ & $\mathrm{OD}=0.67$ & $\mathrm{OD}=1.00$ \\
\hline LLL & 0.053 & 0.062 & 0.072 & 0.080 \\
naïve & 0.002 & 0.011 & 0.020 & 0.030 \\
min & -0.001 & 0.001 & 0.001 & 0.001 \\
$\max$ & -0.001 & -0.002 & -0.002 & -0.001 \\
\hline
\end{tabular}

Table A.2: LLL bias for various OD values, holding CD at zero. Naïve and bounds biases from Section 8.2 are reproduced here for convenience.

Tables A.1 and A.2 show the results in terms of bias at the sample size of 3,000 (second row from the top), along with the comparable results for the naïve estimator and our proposed bounds estimator (third row and below), which are reproduced from Tables 2 and 3 in the main text. Somewhat surprisingly, and contrary to the original findings by LLL based on a much simpler simulation setup, the LLL estimator exhibits substantial bias even when both CD and OD are zero. This suggests that finite-sample performance of the LLL estimator is rather poor when applied to datasets like ours, rendering it an unattractive option for inference.

Next, we contrast the proposed Bayesian inferential approach described in Section A.5 to an alternative method based on the nonparametric bootstrap. We construct the $95 \%$ bootstrap confidence intervals by taking the 2.5 th and 97.5 th percentiles of parameter estimates in 1000 bootstrap draws. For the bounds, we take those percentiles from the lower and upper bound estimates, respectively, to construct confidence intervals that are purported to cover the nonparametric bounds $95 \%$ of the time.

Tables A.3 and A.4 show estimated coverage rates for the 95\% bootstrap confidence intervals at various values of the $\mathrm{CD}$ and $\mathrm{OD}$ parameters. The comparable results for our proposed Bayesian intervals can be found in Tables A.3 and A.4 in the main text. In general, we find that the coverage of the bootstrap intervals is noticeably below that of our proposed method, and the bootstrap coverage rates are 


\begin{tabular}{rrrrr}
\hline $\mathrm{n}$ & $\mathrm{CD}=0.00$ & $\mathrm{CD}=0.33$ & $\mathrm{CD}=0.67$ & $\mathrm{CD}=1.00$ \\
\hline 500 & 0.944 & 0.930 & 0.895 & 0.891 \\
1000 & 0.941 & 0.915 & 0.911 & 0.906 \\
3000 & 0.952 & 0.914 & 0.908 & 0.924 \\
10000 & 0.930 & 0.924 & 0.924 & 0.928 \\
50000 & 0.936 & 0.940 & 0.940 & 0.942 \\
\hline
\end{tabular}

Table A.3: Bootstrap coverage rates for various CD values, holding OD at zero.

\begin{tabular}{rrrrr}
\hline $\mathrm{n}$ & $\mathrm{OD}=0.00$ & $\mathrm{OD}=0.33$ & $\mathrm{OD}=0.67$ & $\mathrm{OD}=1.00$ \\
\hline 500 & 0.944 & 0.954 & 0.949 & 0.950 \\
1000 & 0.941 & 0.960 & 0.956 & 0.959 \\
3000 & 0.952 & 0.942 & 0.952 & 0.944 \\
10000 & 0.930 & 0.952 & 0.950 & 0.958 \\
50000 & 0.936 & 0.948 & 0.944 & 0.946
\end{tabular}

Table A.4: Bootstrap coverage rates for various OD values, holding CD at zero.

substantially below nominal at lower sample sizes and for larger values of the CD parameter. 
Sensitivity Analysis for Discussing Story with Friends (binary)

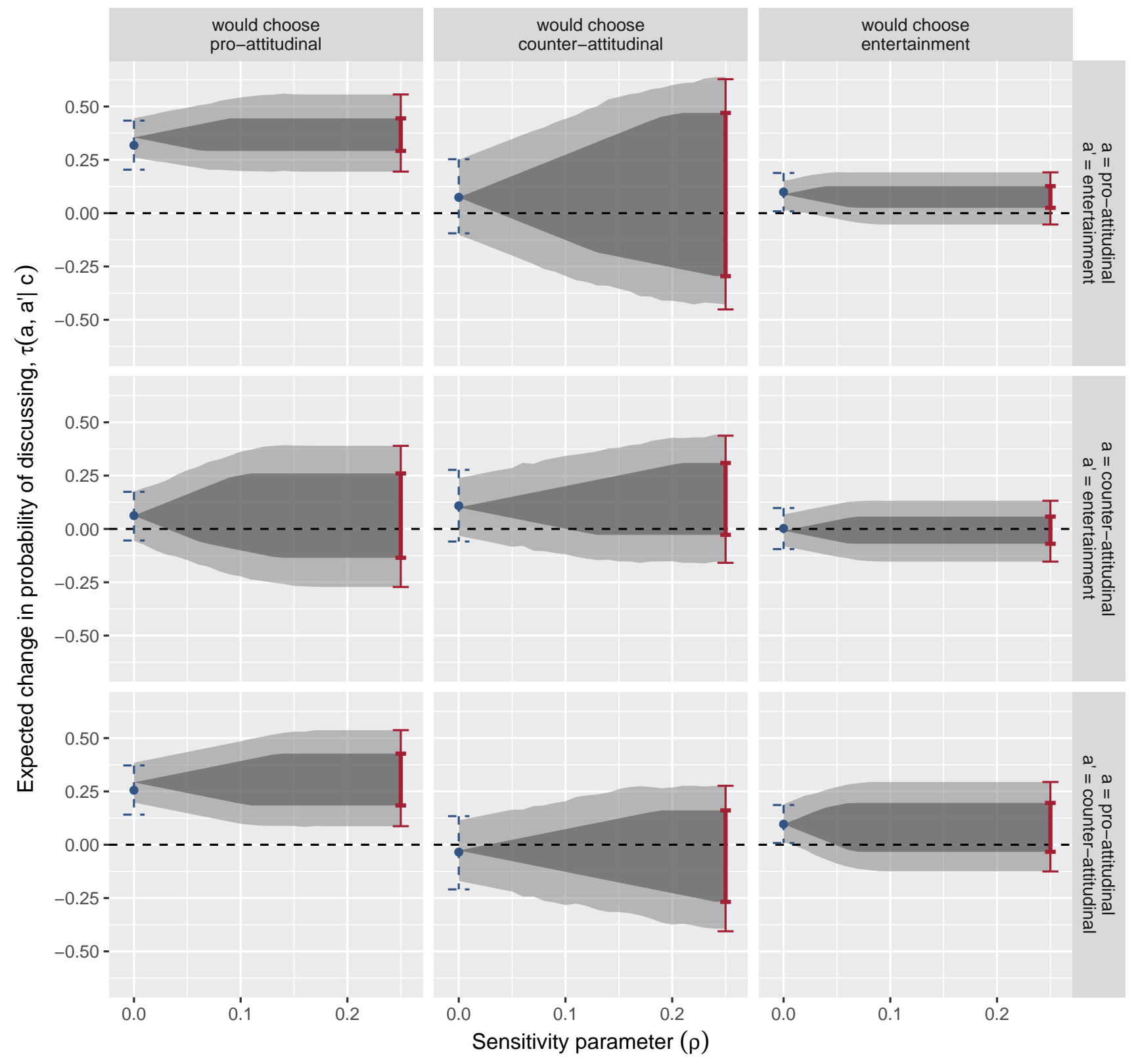

Figure A.1: Sensitivity Analysis for the ACTE of Partisan News Media (Binary Outcome). The plots correspond to the right panel of Figure 2 . See caption for Figure 3 for the explanation of graph elements. 